\title{
Infinite series identities from modular transformation formulas that stem from generalized Eisenstein series
}

\author{
by \\ Sung-Geun Lim (Pohang)
}

1. Introduction. B. C. Berndt [3] has found a general class of Eisenstein series and has computed transformation formulas for them. In [5] he has also converted these transformation formulas into transformation formulas for a large class of functions containing the classical Dedekind eta function. By this process, in [4], [5] he has established a number of interesting infinite series identities, many of which are stated in the Notebooks of Ramanujan [11] or are proved by many authors.

In [8, using the latter transformation formulas (Theorem 2.1), the present author also evaluated many infinite series identities, a number of which are given by other authors including Ramanujan and B. C. Berndt, and many of which are new series or generalizations of series given by other authors. In this paper, we give more general infinite series identities. In Section 3, we obtain a few classes of infinite series for an arbitrary positive integer $c^{\prime}$. A special case of those series (when $c^{\prime}=2$ ) is considered in Section 4. Some of them have already been found by Berndt [4, [5] and other authors. But many of them are new series involving hyperbolic functions and trigonometric functions. In particular, we obtain some elegant symmetric (in $\alpha, \beta)$ identities in Section 4.1.

2. Notation and preliminaries. We basically follow the notation in 5. The branch of the argument for a complex $w$ is defined by $-\pi \leq$ $\arg w<\pi$. We set $e(w)=e^{2 \pi i w}$, and $V \tau=V(\tau)=(a \tau+b) /(c \tau+d)$ always denotes a modular transformation with $c>0$ and $c \equiv 0(\bmod N)$ for every complex $\tau$. Let $r=\left(r_{1}, r_{2}\right)$ and $h=\left(h_{1}, h_{2}\right)$ denote real vectors, and the associated vectors $R$ and $H$ are defined by

2010 Mathematics Subject Classification: Primary 34A25; Secondary 11M36.

Key words and phrases: modular transformation, infinite series, Eisenstein series. 


$$
\begin{aligned}
& R=\left(R_{1}, R_{2}\right)=\left(a r_{1}+c r_{2}, b r_{1}+d r_{2}\right), \\
& H=\left(H_{1}, H_{2}\right)=\left(d h_{1}-b h_{2},-c h_{1}+a h_{2}\right) .
\end{aligned}
$$

For a positive integer $N$, let $\lambda_{N}$ denote the characteristic function of the integers modulo $N$, i.e.,

$$
\lambda_{N}(m)= \begin{cases}1 & \text { if } m \equiv 0(\bmod N) \\ 0 & \text { otherwise }\end{cases}
$$

For a real number $x,[x]$ denotes the greatest integer less than or equal to $x$ and $\{x\}:=x-[x]$. For real $\alpha, x$ and $\operatorname{Re}(s)>1$, let

$$
\psi(x, \alpha, s):=\sum_{n+\alpha>0} \frac{e(n x)}{(n+\alpha)^{s}} .
$$

If $x$ is an integer and $\alpha$ is not an integer, then $\psi(x, \alpha, s)=\zeta(s,\{\alpha\})$, where $\zeta(s, x)$ is the Hurwitz zeta function. The function $\psi(x, \alpha, s)$ can be analytically continued to the entire $s$-plane [2] except for $s=1$ when $x$ is an integer. Let $\mathbb{H}=\{\tau \in \mathbb{C} \mid \operatorname{Im}(\tau)>0\}$, the upper half-plane. For $\tau \in \mathbb{H}$ and an arbitrary complex number $s$, define

$$
A_{N}(\tau, s ; r, h):=\sum_{N m+r_{1}>0} \sum_{n-h_{2}>0} \frac{e\left(N m h_{1}+\left(\left(N m+r_{1}\right) \tau+r_{2}\right)\left(n-h_{2}\right)\right)}{\left(n-h_{2}\right)^{1-s}} .
$$

Let

$$
H_{N}(\tau, s ; r, h):=A_{N}(\tau, s ; r, h)+e(s / 2) A_{N}(\tau, s ;-r,-h) .
$$

It is easy to see that the logarithm of the classical Dedekind eta function is expressed using $H_{N}$. We now state the theorem about transformation formulas for a large class of functions containing the classical Dedekind eta function.

Theorem 2.1 ([5], [8]). Let $Q=\{\tau \in \mathbb{C} \mid \operatorname{Re}(\tau)>-d / c\}, \varrho_{N}=$ $c\left\{R_{2}\right\}-N d\left\{R_{1} / N\right\}$ and $c=N c^{\prime}$. Then for $\tau \in Q$ and all $s$,

$$
\begin{aligned}
& (c \tau+d)^{-s} H_{N}(V \tau, s ; r, h)=H_{N}(\tau, s ; R, H) \\
& -\lambda_{N}\left(r_{1}\right) e\left(-r_{1} h_{1}\right)(c \tau+d)^{-s} \Gamma(s)(-2 \pi i)^{-s} \\
& \quad \times\left(\psi\left(h_{2}, r_{2}, s\right)+e(s / 2) \psi\left(-h_{2},-r_{2}, s\right)\right) \\
& +\lambda_{N}\left(R_{1}\right) e\left(-R_{1} H_{1}\right) \Gamma(s)(-2 \pi i)^{-s}\left(\psi\left(H_{2}, R_{2}, s\right)+e(-s / 2) \psi\left(-H_{2},-R_{2}, s\right)\right) \\
& +(2 \pi i)^{-s} L_{N}(\tau, s ; R, H),
\end{aligned}
$$

where 


$$
\begin{aligned}
& L_{N}(\tau, s ; R, H) \\
& \qquad=\sum_{j=1}^{c^{\prime}} e\left(-H_{1}\left(N j+N\left[R_{1} / N\right]-c\right)-H_{2}\left(\left[R_{2}\right]+1+\left[\left(N j d+\varrho_{N}\right) / c\right]-d\right)\right) \\
& \quad \times \int_{C} u^{s-1} \frac{e^{-(c \tau+d)\left(N j-N\left\{R_{1} / N\right\}\right) u / c}}{e^{-(c \tau+d) u}-e\left(c H_{1}+d H_{2}\right)} \frac{e^{\left\{\left(N j d+\varrho_{N}\right) / c\right\} u}}{e^{u}-e\left(-H_{2}\right)} d u,
\end{aligned}
$$

where $C$ is a loop beginning at $+\infty$, running in the upper half-plane, encircling the origin in the positive direction so that $u=0$ is the only zero of

$$
\left(e^{-(c \tau+d) u}-e\left(c H_{1}+d H_{2}\right)\right)\left(e^{u}-e\left(-H_{2}\right)\right)
$$

lying "inside" the loop, and then returning to $+\infty$ in the lower half-plane. Here, we choose the branch of $u^{s}$ with $0<\arg u<2 \pi$.

REMARK 2.2. Note that after evaluation of $L_{N}(\tau, s ; R, H)$ for an integer $s$, the transformation formula in Theorem 2.1 will be valid for all $\tau \in \mathbb{H}$ by analytic continuation.

For computations of the integral in Theorem 2.1 when $s$ is an integer, it is useful to employ the generating function

$$
\frac{t e^{x t}}{e^{t}-1}=\sum_{n=0}^{\infty} B_{n}(x) \frac{t^{n}}{n !} \quad(|t|<2 \pi)
$$

for Bernoulli polynomials $B_{n}(x), n \geq 0$. The $n$th Bernoulli number $B_{n}$, $n \geq 0$, is defined by $B_{n}=B_{n}(0)$. Put $\bar{B}_{n}(x)=B_{n}(\{x\}), n \geq 0$. Recall that $B_{2 n+1}=0, n \geq 1$, and that $B_{2 n+1}(1 / 2)=0, n \geq 0$. The following formulas are helpful (see [1]):

$$
\begin{aligned}
B_{n}(1-x) & =(-1)^{n} B_{n}(x), \\
\sum_{j=0}^{c-1} B_{n}(j / c+x) & =c^{1-n} B_{n}(c x) .
\end{aligned}
$$

For the zeta function $\zeta(s)$, we know from [7, p. 194] that

$$
\zeta(2 M)=\frac{2^{2 M-1}\left|B_{2 M}\right| \pi^{2 M}}{(2 M) !}, \quad M>0,
$$

and

$$
\zeta(1-2 M)=-\frac{B_{2 M}}{2 M}, \quad M>0 .
$$

For brevity, we let

$$
\begin{aligned}
& D_{1}\left(h_{2}, r_{2}, s\right):=-(c \tau+d)^{-s} \Gamma(s)(-2 \pi i)^{-s} \\
& \times\left(\psi\left(h_{2}, r_{2}, s\right)+e(s / 2) \psi\left(-h_{2},-r_{2}, s\right)\right), \\
& D_{2}\left(H_{2}, R_{2}, s\right):=\Gamma(s)(-2 \pi i)^{-s}\left(\psi\left(H_{2}, R_{2}, s\right)+e(-s / 2) \psi\left(-H_{2},-R_{2}, s\right)\right),
\end{aligned}
$$


and

$$
\mathcal{Z}_{ \pm}(s, x):=\zeta(s, x) \pm \zeta(s, 1-x)
$$

3. Several classes of infinite series. In this section, we study a more general series than those in [8]. Let $N$ be a positive integer, and let $r_{1}$ and $r_{2}$ be arbitrary real numbers. For any integer $n$, put

$$
r=\left(r_{1}, r_{2} / c\right), \quad h=(0,0), \quad s=-n .
$$

Let $V$ denote a modular transformation corresponding to

$$
\left(\begin{array}{cc}
1 & -1 \\
c & -c+1
\end{array}\right),
$$

where $c=N c^{\prime}$ for $c^{\prime}>0$. Let $z=c \tau+1-c$. Then $V \tau=1 / c-1 / c z$. The condition $\tau \in Q$ gives $\operatorname{Re}(z)>0$ and $z \in \mathbb{H}$. We also have

$$
R_{1}=r_{1}+r_{2}, \quad R_{2}=-r_{1}-r_{2}+r_{2} / c .
$$

We consider four cases of $N, r_{1}$ and $r_{2}$ and, by the same process as in [8], deduce (i)-(iv) below.

(i) If $N \nmid r_{1}$, then

$$
\begin{aligned}
H_{N}(V \tau, & -2 n ; r, 0) \\
= & \sum_{k=1}^{\infty} \frac{\cosh \left(\frac{\pi i k}{c^{\prime}}\left(\frac{2\left(r_{1}+r_{2}\right)}{N}-2\left[\frac{r_{1}}{N}\right]-1+\left(1-2\left\{\frac{r_{1}}{N}\right\}\right) \frac{1}{z}\right)\right)}{k^{2 n+1} \sinh \left(\frac{\pi i k}{c^{\prime}}\left(\frac{1}{z}-1\right)\right)}, \\
H_{N}(V \tau, & -2 n-1 ; r, 0) \\
= & \sum_{k=1}^{\infty} \frac{\sinh \left(\frac{\pi i k}{c^{\prime}}\left(\frac{2\left(r_{1}+r_{2}\right)}{N}-2\left[\frac{r_{1}}{N}\right]-1+\left(1-2\left\{\frac{r_{1}}{N}\right\}\right) \frac{1}{z}\right)\right)}{k^{2 n+2} \sinh \left(\frac{\pi i k}{c^{\prime}}\left(\frac{1}{z}-1\right)\right)} .
\end{aligned}
$$

(ii) If $N \mid r_{1}$, then

$$
\begin{gathered}
H_{N}(V \tau,-2 n ; r, 0)=2 \sum_{k=1}^{\infty} \frac{\cos \left(2 \pi r_{2} k / c\right)}{k^{2 n+1}\left(e^{\frac{2 \pi i k}{c^{\prime}}\left(\frac{1}{z}-1\right)}-1\right)}, \\
H_{N}(V \tau,-2 n-1 ; r, 0)=2 i \sum_{k=1}^{\infty} \frac{\sin \left(2 \pi r_{2} k / c\right)}{k^{2 n+2}\left(e^{\frac{2 \pi i k}{c^{\prime}}\left(\frac{1}{z}-1\right)}-1\right)} .
\end{gathered}
$$

(iii) If $N \nmid R_{1}=r_{1}+r_{2}$, then

$$
\begin{aligned}
H_{N}(\tau, & -2 n ; R, 0) \\
= & \sum_{k=1}^{\infty} \frac{\cosh \left(\frac{\pi i k}{c^{\prime}}\left(\frac{-2 r_{1}}{N}+2\left[\frac{r_{1}+r_{2}}{N}\right]+1+\left(2\left\{\frac{r_{1}+r_{2}}{N}\right\}-1\right) z\right)\right)}{k^{2 n+1} \sinh \left(\frac{\pi i k}{c^{\prime}}(1-z)\right)},
\end{aligned}
$$




$$
\begin{aligned}
H_{N}(\tau, & -2 n-1 ; R, 0) \\
= & \sum_{k=1}^{\infty} \frac{\sinh \left(\frac{\pi i k}{c^{\prime}}\left(\frac{-2 r_{1}}{N}+2\left[\frac{r_{1}+r_{2}}{N}\right]+1+\left(2\left\{\frac{r_{1}+r_{2}}{N}\right\}-1\right) z\right)\right)}{k^{2 n+2} \sinh \left(\frac{\pi i k}{c^{\prime}}(1-z)\right)} .
\end{aligned}
$$

(iv) If $N \mid R_{1}=r_{1}+r_{2}$, then

$$
\begin{gathered}
H_{N}(\tau,-2 n ; R, 0)=2 \sum_{k=1}^{\infty} \frac{\cos \left(2 \pi r_{2} k / c\right)}{k^{2 n+1}\left(e^{\frac{2 \pi i k}{c^{\prime}}(1-z)}-1\right)}, \\
H_{N}(\tau,-2 n-1 ; R, 0)=2 i \sum_{k=1}^{\infty} \frac{\sin \left(2 \pi r_{2} k / c\right)}{k^{2 n+2}\left(e^{\frac{2 \pi i k}{c^{\prime}}(1-z)}-1\right)} .
\end{gathered}
$$

Next, by the residue theorem, we find that

$$
\begin{aligned}
& L_{N}(\tau,-n ; R, 0) \\
& \quad=2 \pi i \sum_{j=1}^{c^{\prime}} \sum_{k=0}^{n+2} \frac{B_{k}\left(\frac{j-\left\{\left(r_{1}+r_{2}\right) / N\right\}}{c^{\prime}}\right) \bar{B}_{n+2-k}\left(\frac{j+\varrho_{N} / N}{c^{\prime}}\right)}{k !(n+2-k) !}(-z)^{k-1} .
\end{aligned}
$$

Applying (3.1)-(3.9) to Theorem 2.1, we obtain a general class of infinite series.

3.1. Case of $N \nmid r_{1}$ and $N \nmid\left(r_{1}+r_{2}\right)$. In this case, $\lambda_{N}\left(r_{1}\right)=$ $\lambda_{N}\left(R_{1}\right)=0$, and by Theorem 2.1. we see that, for any integer $n$ and $z \in \mathbb{H}$ with $\operatorname{Re}(z)>0$,

$$
z^{n} H_{N}(V \tau,-n ; r, 0)=H_{N}(\tau,-n ; R, 0)+(2 \pi i)^{n} L_{N}(\tau,-n ; R, 0) .
$$

Putting (3.1), (3.2), 3.5), (3.6) and 3.9) into (3.10) and employing Remark 2.2, we find that, for any integer $n$ and $z \in \mathbb{H}$,

$$
\begin{aligned}
& z^{2 n} \sum_{k=1}^{\infty} \frac{\cosh \left(\frac{\pi i k}{c^{\prime}}\left(\frac{2\left(r_{1}+r_{2}\right)}{N}-2\left[\frac{r_{1}}{N}\right]-1+\left(1-2\left\{\frac{r_{1}}{N}\right\}\right) \frac{1}{z}\right)\right)}{k^{2 n+1} \sinh \left(\frac{\pi i k}{c^{\prime}}\left(\frac{1}{z}-1\right)\right)} \\
= & \sum_{k=1}^{\infty} \frac{\cosh \left(\frac{\pi i k}{c^{\prime}}\left(\frac{-2 r_{1}}{N}+2\left[\frac{r_{1}+r_{2}}{N}\right]+1+\left(2\left\{\frac{r_{1}+r_{2}}{N}\right\}-1\right) z\right)\right)}{k^{2 n+1} \sinh \left(\frac{\pi i k}{c^{\prime}}(1-z)\right)} \\
& +(2 \pi i)^{2 n+1} \sum_{j=1}^{c^{\prime}} \sum_{k=0}^{2 n+2} \frac{B_{k}\left(\frac{j-\left\{\left(r_{1}+r_{2}\right) / N\right\}}{c^{\prime}}\right) \bar{B}_{2 n+2-k}\left(\frac{j+\varrho_{N} / N}{c^{\prime}}\right)}{k !(2 n+2-k) !}(-z)^{k-1},
\end{aligned}
$$

and

$$
z^{2 n+1} \sum_{k=1}^{\infty} \frac{\sinh \left(\frac{\pi i k}{c^{\prime}}\left(\frac{2\left(r_{1}+r_{2}\right)}{N}-2\left[\frac{r_{1}}{N}\right]-1+\left(1-2\left\{\frac{r_{1}}{N}\right\}\right) \frac{1}{z}\right)\right)}{k^{2 n+2} \sinh \left(\frac{\pi i k}{c^{\prime}}\left(\frac{1}{z}-1\right)\right)}
$$




$$
\begin{aligned}
= & \sum_{k=1}^{\infty} \frac{\sinh \left(\frac{\pi i k}{c^{\prime}}\left(\frac{-2 r_{1}}{N}+2\left[\frac{r_{1}+r_{2}}{N}\right]+1+\left(2\left\{\frac{r_{1}+r_{2}}{N}\right\}-1\right) z\right)\right)}{k^{2 n+2} \sinh \left(\frac{\pi i k}{c^{\prime}}(1-z)\right)} \\
& +(2 \pi i)^{2 n+2} \sum_{j=1}^{c^{\prime}} \sum_{k=0}^{2 n+3} \frac{B_{k}\left(\frac{j-\left\{\left(r_{1}+r_{2}\right) / N\right\}}{c^{\prime}}\right) \bar{B}_{2 n+3-k}\left(\frac{j+\varrho_{N} / N}{c^{\prime}}\right)}{k !(2 n+3-k) !}(-z)^{k-1} .
\end{aligned}
$$

Theorem 3.1. Let $\alpha, \beta>0$ with $\alpha \beta=\pi^{2}$. Suppose that $r$, $r^{\prime}$ are real numbers such that $r, r+r^{\prime}$ are not integers. Then, for any integer $n$ and for any positive integer $c^{\prime}$,

$$
\begin{aligned}
\alpha^{-n} \sum_{k=1}^{\infty} & \frac{\cosh \left(\left(2\left(r+r^{\prime}\right)-2[r]-1\right) \pi i k / c^{\prime}+(1-2\{r\}) \alpha k / c^{\prime}\right)}{k^{2 n+1} \sinh \left((\alpha-i \pi) k / c^{\prime}\right)} \\
= & (-\beta)^{-n} \sum_{k=1}^{\infty} \frac{\cosh \left(\left(-2 r+2\left[r+r^{\prime}\right]+1\right) \pi i k / c^{\prime}+\left(1-2\left\{r+r^{\prime}\right\}\right) \beta k / c^{\prime}\right)}{k^{2 n+1} \sinh \left((\beta+i \pi) k / c^{\prime}\right)} \\
& -2^{2 n+1} \sum_{j=1}^{c^{\prime}} \sum_{k=0}^{2 n+2} \frac{B_{k}\left(\frac{j-\left\{r+r^{\prime}\right\}}{c^{\prime}}\right) \bar{B}_{2 n+2-k}\left(\frac{j+\varrho_{N} / N}{c^{\prime}}\right)}{k !(2 n+2-k) !}(-\pi i)^{k} \alpha^{n-k+1},
\end{aligned}
$$

and

$$
\begin{aligned}
& \alpha^{-n-1 / 2} \sum_{k=1}^{\infty} \frac{\sinh \left(\left(2\left(r+r^{\prime}\right)-2[r]-1\right) \pi i k / c^{\prime}+(1-2\{r\}) \alpha k / c^{\prime}\right)}{k^{2 n+2} \sinh \left((\alpha-i \pi) k / c^{\prime}\right)} \\
& =(-\beta)^{-n-1 / 2} \sum_{k=1}^{\infty} \frac{\sinh \left(\left(-2 r+2\left[r+r^{\prime}\right]+1\right) \pi i k / c^{\prime}+\left(1-2\left\{r+r^{\prime}\right\}\right) \beta k / c^{\prime}\right)}{k^{2 n+2} \sinh \left((\beta+i \pi) k / c^{\prime}\right)} \\
& \quad-2^{2 n+2} \sum_{j=1}^{c^{\prime}} \sum_{k=0}^{2 n+3} \frac{B_{k}\left(\frac{j-\left\{r+r^{\prime}\right\}}{c^{\prime}}\right) \bar{B}_{2 n+3-k}\left(\frac{j+\varrho_{N} / N}{c^{\prime}}\right)}{k !(2 n+3-k) !}(-\pi i)^{k} \alpha^{n-k+3 / 2} .
\end{aligned}
$$

Proof. Put $z=\pi i / \alpha$ in (3.11) and (3.12), and replace $r_{1} / N$ and $r_{2} / N$ by $r$ and $r^{\prime}$, respectively. Then the desired results follow.

We now assume that $r$ is a real number with $0<r<1$ and $r^{\prime}=0$ in Theorem 3.1. Then it is easy see that

$$
\left\{\frac{j+\varrho_{N} / N}{c^{\prime}}\right\}=\left\{\frac{j+c^{\prime}\{-N r\}+N c^{\prime} r-r}{c^{\prime}}\right\}=\left\{\frac{j-r}{c^{\prime}}\right\} .
$$

Thus, from Theorem 3.1 we obtain the following theorem.

TheOREM 3.2. Let $\alpha, \beta>0$ with $\alpha \beta=\pi^{2}$. Let $r$ be any real number with $0<r<1$. Then, for any integer $n$ and for any positive integer $c^{\prime}$, 
$(3.13)$

$$
\begin{aligned}
\alpha^{-n} \sum_{k=1}^{\infty} & \frac{\cosh \left((1-2 r)(\alpha-i \pi) k / c^{\prime}\right)}{k^{2 n+1} \sinh \left((\alpha-i \pi) k / c^{\prime}\right)} \\
= & (-\beta)^{-n} \sum_{k=1}^{\infty} \frac{\cosh \left((1-2 r)(\beta+i \pi) k / c^{\prime}\right)}{k^{2 n+1} \sinh \left((\beta+i \pi) k / c^{\prime}\right)} \\
& -2^{2 n+1} \sum_{j=1}^{c^{\prime}} \sum_{k=0}^{2 n+2} \frac{B_{k}\left(\frac{j-r}{c^{\prime}}\right) B_{2 n+2-k}\left(\frac{j-r}{c^{\prime}}\right)}{k !(2 n+2-k) !}(-\pi i)^{k} \alpha^{n-k+1},
\end{aligned}
$$

and

$$
\begin{aligned}
\alpha^{-n-1 / 2} & \sum_{k=1}^{\infty} \frac{\sinh \left((1-2 r)(\alpha-i \pi) k / c^{\prime}\right)}{k^{2 n+2} \sinh \left((\alpha-i \pi) k / c^{\prime}\right)} \\
= & (-\beta)^{-n-1 / 2} \sum_{k=1}^{\infty} \frac{\sinh \left((1-2 r)(\beta+i \pi) k / c^{\prime}\right)}{k^{2 n+2} \sinh \left((\beta+i \pi) k / c^{\prime}\right)} \\
& -2^{2 n+2} \sum_{j=1}^{c^{\prime}} \sum_{k=0}^{2 n+3} \frac{B_{k}\left(\frac{j-r}{c^{\prime}}\right) B_{2 n+3-k}\left(\frac{j-r}{c^{\prime}}\right)}{k !(2 n+3-k) !}(-\pi i)^{k} \alpha^{n-k+3 / 2} .
\end{aligned}
$$

Corollary 3.3 .

$$
\begin{aligned}
\alpha^{-n} \sum_{k=1}^{\infty} \frac{\operatorname{csch}\left((\alpha-i \pi) k / c^{\prime}\right)}{k^{2 n+1}} \\
=(-\beta)^{-n} \sum_{k=1}^{\infty} \frac{\operatorname{csch}\left((\beta+i \pi) k / c^{\prime}\right)}{k^{2 n+1}} \\
\quad-2^{2 n+1} \sum_{j=1}^{c^{\prime}} \sum_{k=0}^{2 n+2} \frac{B_{k}\left(\frac{2 j-1}{2 c^{\prime}}\right) B_{2 n+2-k}\left(\frac{2 j-1}{2 c^{\prime}}\right)}{k !(2 n+2-k) !}(-\pi i)^{k} \alpha^{n-k+1} .
\end{aligned}
$$

Proof. Put $r=1 / 2$ in 3.13 .

Corollary 3.4.

$$
\begin{aligned}
& \sum_{k=1}^{\infty} \frac{\cosh \left(\pi k / c^{\prime}\right) \sin \left(\pi k / c^{\prime}\right)}{k^{4 n+1}\left(\cosh \left(2 \pi k / c^{\prime}\right)-\cos \left(2 \pi k / c^{\prime}\right)\right)} \\
& =\frac{(2 \pi)^{4 n+1}}{2} \sum_{j=1}^{c^{\prime}} \sum_{k=0}^{4 n+2} \frac{B_{k}\left(\frac{2 j-1}{2 c^{\prime}}\right) B_{4 n+2-k}\left(\frac{2 j-1}{2 c^{\prime}}\right)}{k !(4 n+2-k) !}(-i)^{k-1} .
\end{aligned}
$$

Proof. Let $\alpha=\beta=\pi$ in Corollary 3.3 and replace $n$ by $2 n$. Use

$$
\operatorname{csch}(x-i y)-\operatorname{csch}(x+i y)=\frac{2 i \cosh (x) \sin (y)}{\cosh (2 x)-\cos (2 y)} .
$$


Corollary 3.5 .

$$
\begin{aligned}
& \sum_{k=1}^{\infty} \frac{\sinh \left(\pi k / c^{\prime}\right) \cos \left(\pi k / c^{\prime}\right)}{k^{4 n+3}\left(\cosh \left(2 \pi k / c^{\prime}\right)-\cos \left(2 \pi k / c^{\prime}\right)\right)} \\
& =-\frac{(2 \pi)^{4 n+3}}{2} \sum_{j=1}^{c^{\prime}} \sum_{k=0}^{4 n+4} \frac{B_{k}\left(\frac{2 j-1}{2 c^{\prime}}\right) B_{4 n+4-k}\left(\frac{2 j-1}{2 c^{\prime}}\right)}{k !(4 n+4-k) !}(-i)^{k} .
\end{aligned}
$$

Proof. Let $\alpha=\beta=\pi$ in Corollary 3.3 and replace $n$ by $2 n+1$. Use

$$
\operatorname{csch}(x-i y)+\operatorname{csch}(x+i y)=\frac{2 \sinh (x) \cos (y)}{\cosh (2 x)-\cos (2 y)}
$$

Corollary 3.6.

$$
\alpha \sum_{k=1}^{\infty} k \operatorname{csch}\left((\alpha-i \pi) k / c^{\prime}\right)+\beta \sum_{k=1}^{\infty} k \operatorname{csch}\left((\beta+i \pi) k / c^{\prime}\right)=-c^{\prime} / 2 .
$$

Proof. Put $n=-1$ in Corollary 3.3 .

Corollary 3.7.

$$
\sum_{k=1}^{\infty} \frac{k \sinh \left(\pi k / c^{\prime}\right) \cos \left(\pi k / c^{\prime}\right)}{\cosh \left(2 \pi k / c^{\prime}\right)-\cos \left(2 \pi k / c^{\prime}\right)}=-\frac{c^{\prime}}{2 \pi}
$$

Proof. Put $n=-1$ in Corollary 3.5 .

Corollary 3.8 .

$$
\sum_{k=1}^{\infty} \frac{\operatorname{csch}\left((\alpha-i \pi) k / c^{\prime}\right)}{k}=\sum_{k=1}^{\infty} \frac{\operatorname{csch}\left((\beta+i \pi) k / c^{\prime}\right)}{k}-\frac{\beta-\alpha}{12 c^{\prime}}+\frac{\pi i}{6}\left(c^{\prime}-\frac{1}{c^{\prime}}\right) .
$$

Proof. Put $n=0$ in Corollary 3.3. It is easy to see that

$$
\sum_{j=1}^{c^{\prime}} B_{1}\left(\frac{2 j-1}{2 c^{\prime}}\right)^{2}=\sum_{j=1}^{c^{\prime}}\left(\frac{2 j-1}{2 c^{\prime}}-\frac{1}{2}\right)^{2}=\frac{1}{12}\left(c^{\prime}-\frac{1}{c^{\prime}}\right) \text {. }
$$

Corollary 3.9 .

$$
\sum_{k=1}^{\infty} \frac{\cosh \left(\pi k / c^{\prime}\right) \sin \left(\pi k / c^{\prime}\right)}{k\left(\cosh \left(2 \pi k / c^{\prime}\right)-\cos \left(2 \pi k / c^{\prime}\right)\right)}=\frac{\pi}{12}\left(c^{\prime}-\frac{1}{c^{\prime}}\right) .
$$

Proof. Let $\alpha=\beta=\pi$ in Corollary 3.8 . 
Corollary 3.10 .

$$
\begin{aligned}
\alpha^{-n-1 / 2} & \sum_{k=1}^{\infty} \frac{\operatorname{sech}\left((\alpha-i \pi) \frac{k}{2 c^{\prime}}\right)}{k^{2 n+2}} \\
= & (-\beta)^{-n-1 / 2} \sum_{k=1}^{\infty} \frac{\operatorname{sech}\left((\beta+i \pi) \frac{k}{2 c^{\prime}}\right)}{k^{2 n+2}} \\
& -2^{2 n+3} \sum_{j=1}^{c^{\prime}} \sum_{k=0}^{2 n+3} \frac{B_{k}\left(\frac{4 j-1}{4 c^{\prime}}\right) B_{2 n+3-k}\left(\frac{4 j-1}{4 c^{\prime}}\right)}{k !(2 n+3-k) !}(-\pi i)^{k} \alpha^{n-k+3 / 2} .
\end{aligned}
$$

Proof. Let $r=1 / 4$ in 3.14.

Corollary 3.11 .

$$
\begin{aligned}
\alpha^{1 / 2}\left(\sum_{k=1}^{\infty} \operatorname{sech}\left((\alpha-i \pi) \frac{k}{2 c^{\prime}}\right)\right. & \left.+\frac{1}{2}\right) \\
= & (-\beta)^{1 / 2}\left(\sum_{k=1}^{\infty} \operatorname{sech}\left((\beta+i \pi) \frac{k}{2 c^{\prime}}\right)+\frac{1}{2}\right) .
\end{aligned}
$$

Proof. Let $n=-1$ in Corollary 3.10. We see that

$$
-2 \sum_{j=1}^{c^{\prime}} \sum_{k=0}^{1} \frac{B_{k}\left(\frac{4 j-1}{4 c^{\prime}}\right) B_{1-k}\left(\frac{4 j-1}{4 c^{\prime}}\right)}{k !(1-k) !}(-\pi i)^{k} \alpha^{-k+1 / 2}=-\frac{1}{2} \alpha^{1 / 2}+\frac{1}{2}(-\beta)^{1 / 2} .
$$

3.2. Case of $N \mid r_{1}$ and $N \nmid\left(r_{1}+r_{2}\right)$. By Theorem 2.1, for any integer $n$ and $z \in \mathbb{H}$ with $\operatorname{Re}(z)>0$,

$$
\begin{aligned}
z^{n} H_{N}(V \tau,-n ; r, 0)= & H_{N}(\tau,-n ; R, 0)+(2 \pi i)^{n} L_{N}(\tau,-n ; R, 0) \\
& +\lim _{s \rightarrow-n} D_{1}\left(0, r_{2} / c, s\right)
\end{aligned}
$$

where $D_{1}\left(0, r_{2} / c, s\right)$ is defined in Section 2. Since

$$
\begin{aligned}
\frac{\varrho_{N}}{N} & =c^{\prime}\left\{-r_{2}+\frac{r_{2}}{N c^{\prime}}\right\}+\left(N c^{\prime}-1\right)\left\{\frac{r_{2}}{N}\right\} \\
& =-c^{\prime}\left[-r_{2}+\frac{r_{2}}{N c^{\prime}}\right]-\left(N c^{\prime}-1\right)\left[\frac{r_{2}}{N}\right],
\end{aligned}
$$

we find that

$$
\left\{\frac{j+\frac{\varrho_{N}}{N}}{c^{\prime}}\right\}=\left\{\frac{j-c^{\prime}\left[-r_{2}+\frac{r_{2}}{N c^{\prime}}\right]-N c^{\prime}\left[\frac{r_{2}}{N}\right]+\left[\frac{r_{2}}{N}\right]}{c^{\prime}}\right\}=\left\{\frac{j+\left[\frac{r_{2}}{N}\right]}{c^{\prime}}\right\} .
$$

Recall the notation of $\mathcal{Z}_{ \pm}(s, x)$ defined in (2.6), i.e.,

$$
\mathcal{Z}_{ \pm}(s, x):=\zeta(s, x) \pm \zeta(s, 1-x)
$$


Then, using Lemma 2.2 in [8] and employing Remark 2.2, we have, for any $z \in \mathbb{H}$,

$$
z^{2 n} \sum_{k=1}^{\infty} \frac{2 \cos \left(2 \pi r_{2} k / c\right)}{k^{2 n+1}\left(e^{\frac{2 \pi i k}{c^{\prime}}\left(\frac{1}{z}-1\right)}-1\right)}
$$

$$
=\sum_{k=1}^{\infty} \frac{\cosh \left(\frac{\pi i k}{c^{\prime}}\left(2\left[\frac{r_{2}}{N}\right]+1+\left(2\left\{\frac{r_{2}}{N}\right\}-1\right) z\right)\right)}{k^{2 n+1} \sinh \left(\frac{\pi i k}{c^{\prime}}(1-z)\right)}
$$

$$
+(2 \pi i)^{2 n+1} \sum_{j=1}^{c^{\prime}} \sum_{k=0}^{2 n+2} \frac{B_{k}\left(\frac{j-\left\{r_{2} / N\right\}}{c^{\prime}}\right) \bar{B}_{2 n+2-k}\left(\frac{j+\left[r_{2} / N\right]}{c^{\prime}}\right)}{k !(2 n+2-k) !}(-z)^{k-1}+J_{0}(n),
$$

where

$$
J_{0}(n):= \begin{cases}-(2 \pi i z)^{2 n}(-2 n-1) ! \mathcal{Z}_{+}\left(-2 n,\left\{r_{2} / c\right\}\right) & \text { if } n<0 \\ \log \left(1-e^{-2 \pi i r_{2} / c}\right) & \text { if } n=0 \\ -z^{2 n} \psi\left(-r_{2} / c, 0,2 n+1\right) & \text { if } n>0\end{cases}
$$

and

$$
\begin{aligned}
& \text { 3.16) } z^{2 n+1} \sum_{k=1}^{\infty} \frac{2 i \sin \left(2 \pi r_{2} k / c\right)}{k^{2 n+2}\left(e^{\frac{2 \pi i k}{c^{\prime}}\left(\frac{1}{z}-1\right)}-1\right)} \\
& =\sum_{k=1}^{\infty} \frac{\sinh \left(\frac{\pi i k}{c^{\prime}}\left(2\left[\frac{r_{2}}{N}\right]+1+\left(2\left\{\frac{r_{2}}{N}\right\}-1\right) z\right)\right)}{k^{2 n+2} \sinh \left(\frac{\pi i k}{c^{\prime}}(1-z)\right)} \\
& +(2 \pi i)^{2 n+2} \sum_{j=1}^{c^{\prime}} \sum_{k=0}^{2 n+3} \frac{B_{k}\left(\frac{j-\left\{r_{2} / N\right\}}{c^{\prime}}\right) \bar{B}_{2 n+3-k}\left(\frac{j+\left[r_{2} / N\right]}{c^{\prime}}\right)}{k !(2 n+3-k) !}(-z)^{k-1}+\mathcal{J}_{0}(n),
\end{aligned}
$$

where

$$
\mathcal{J}_{0}(n):= \begin{cases}(2 \pi i z)^{2 n+1}(-2 n-2) ! \mathcal{Z}_{-}\left(-2 n-1,\left\{r_{2} / c\right\}\right) & \text { if } n<-1, \\ (2 \pi i z)^{-1}\left(\pi \cot \left(\pi\left\{r_{2} / c\right\}\right)-\pi i\right) & \text { if } n=-1, \\ z^{2 n+1} \psi\left(-r_{2} / c, 0,2 n+2\right) & \text { if } n \geq 0 .\end{cases}
$$

Theorem 3.12. Let $\alpha, \beta>0$ with $\alpha \beta=\pi^{2}$. Let $r$ be any real number which is not an integer. Then for any integer $n$ and for any positive integer $c^{\prime}$,

$$
\begin{aligned}
& \alpha^{-n} \sum_{k=1}^{\infty} \frac{2 \cos \left(2 \pi r k / c^{\prime}\right)}{k^{2 n+1}\left(e^{(\alpha-i \pi) 2 k / c^{\prime}}-1\right)} \\
&=(-\beta)^{-n} \sum_{k=1}^{\infty} \frac{\cosh \left((2[r]+1) \pi i k / c^{\prime}+(1-2\{r\}) \beta k / c^{\prime}\right)}{k^{2 n+1} \sinh \left((\beta+i \pi) k / c^{\prime}\right)}
\end{aligned}
$$




$$
-2^{2 n+1} \sum_{j=1}^{c^{\prime}} \sum_{k=0}^{2 n+2} \frac{B_{k}\left(\frac{j-\{r\}}{c^{\prime}}\right) \bar{B}_{2 n+2-k}\left(\frac{j+[r]}{c^{\prime}}\right)}{k !(2 n+2-k) !}(-\pi i)^{k} \alpha^{n-k+1}+J_{1}(n),
$$

where

$$
J_{1}(n):= \begin{cases}-(-4 \beta)^{n}(-2 n-1) ! \mathcal{Z}_{+}\left(-2 n,\left\{r / c^{\prime}\right\}\right) & \text { if } n<0 \\ \log \left(1-e^{-2 \pi i r / c^{\prime}}\right) & \text { if } n=0 \\ -\alpha^{-n} \psi\left(-r / c^{\prime}, 0,2 n+1\right) & \text { if } n>0 .\end{cases}
$$

Proof. Put $z=\pi i / \alpha$ and $r_{2} / N=r$ in (3.15.

Theorem 3.13. Let $\alpha, \beta>0$ with $\alpha \beta=\pi^{2}$. Let $r$ be any real number that is not an integer. Then for any integer $n$ and for any positive integer $c^{\prime}$,

$$
\begin{aligned}
\alpha^{-n-1 / 2} & \sum_{k=1}^{\infty} \frac{2 i \sin \left(2 \pi r k / c^{\prime}\right)}{k^{2 n+2}\left(e^{(\alpha-i \pi) 2 k / c^{\prime}}-1\right)} \\
= & (-\beta)^{-n-1 / 2} \sum_{k=1}^{\infty} \frac{\sinh \left((2[r]+1) \pi i k / c^{\prime}+(1-2\{r\}) \beta k / c^{\prime}\right)}{k^{2 n+2} \sinh \left((\beta+i \pi) k / c^{\prime}\right)} \\
& -2^{2 n+2} \sum_{j=1}^{c^{\prime}} \sum_{k=0}^{2 n+3} \frac{B_{k}\left(\frac{j-\{r\}}{c^{\prime}}\right) \bar{B}_{2 n+3-k}\left(\frac{j+[r]}{c^{\prime}}\right)}{k !(2 n+3-k) !}(-\pi i)^{k} \alpha^{n-k+3 / 2}+\mathcal{J}_{1}(n),
\end{aligned}
$$

where

$$
\mathcal{J}_{1}(n):= \begin{cases}(-4 \beta)^{n+1 / 2}(-2 n-2) ! \mathcal{Z}_{-}\left(-2 n-1,\left\{r / c^{\prime}\right\}\right) & \text { if } n<-1, \\ -\frac{i}{2} \alpha^{1 / 2} \cot \left(\pi\left\{r / c^{\prime}\right\}\right)-\frac{1}{2} \alpha^{1 / 2} & \text { if } n=-1, \\ \alpha^{-n-1 / 2} \psi\left(-r / c^{\prime}, 0,2 n+2\right) & \text { if } n \geq 0 .\end{cases}
$$

Proof. Put $z=\pi i / \alpha$ and $r_{2} / N=r$ in (3.16).

Proposition 3.14. Let $\alpha, \beta>0$ with $\alpha \beta=\pi^{2}$. Then for any integer $n$ and any positive integer $c^{\prime}$,

$$
\begin{aligned}
\alpha^{-n} & \sum_{k=1}^{\infty} \frac{2 \cos \left(\pi k / c^{\prime}\right)}{k^{2 n+1}\left(e^{(\alpha-i \pi) 2 k / c^{\prime}}-1\right)} \\
= & (-\beta)^{-n} \sum_{k=1}^{\infty} \frac{\cos \left(\pi k / c^{\prime}\right)}{k^{2 n+1} \sinh \left((\beta+i \pi) k / c^{\prime}\right)} \\
& \quad-2^{2 n+1} \sum_{j=1}^{c^{\prime}} \sum_{k=0}^{2 n+2} \frac{B_{k}\left(\frac{2 j-1}{2 c^{\prime}}\right) \bar{B}_{2 n+2-k}\left(\frac{j}{c^{\prime}}\right)}{k !(2 n+2-k) !}(-\pi i)^{k} \alpha^{n-k+1}+J_{2}(n),
\end{aligned}
$$

where

$$
J_{2}(n):= \begin{cases}-(-4 \beta)^{n}(-2 n-1) ! \mathcal{Z}_{+}\left(-2 n, 1 / 2 c^{\prime}\right) & \text { if } n<0 \\ \log \left(1-e^{-\pi i / c^{\prime}}\right) & \text { if } n=0, \\ -\alpha^{-n} \psi\left(-1 / 2 c^{\prime}, 0,2 n+1\right) & \text { if } n>0 .\end{cases}
$$


Proof. Put $r=1 / 2$ in Theorem 3.12 .

Proposition 3.15. Let $\alpha, \beta>0$ with $\alpha \beta=\pi^{2}$. Then for any integer $n$ and any positive integer $c^{\prime}$,

$$
\begin{aligned}
\alpha^{-n-1 / 2} \sum_{k=1}^{\infty} \frac{2 \sin \left(\pi k / c^{\prime}\right)}{k^{2 n+2}\left(e^{(\alpha-i \pi) 2 k / c^{\prime}}-1\right)} \\
=(-\beta)^{-n-1 / 2} \sum_{k=1}^{\infty} \frac{\sin \left(\pi k / c^{\prime}\right)}{k^{2 n+2} \sinh \left((\beta+i \pi) k / c^{\prime}\right)} \\
\quad+i 2^{2 n+2} \sum_{j=1}^{c^{\prime}} \sum_{k=0}^{2 n+3} \frac{B_{k}\left(\frac{2 j-1}{2 c^{\prime}}\right) \bar{B}_{2 n+3-k}\left(\frac{j}{c^{\prime}}\right)}{k !(2 n+3-k) !}(-\pi i)^{k} \alpha^{n-k+3 / 2}+\mathcal{J}_{2}(n),
\end{aligned}
$$

where

$$
\mathcal{J}_{2}(n):= \begin{cases}(-1)^{n}(4 \beta)^{n+1 / 2}(-2 n-2) ! \mathcal{Z}_{-}\left(-2 n-1,1 / 2 c^{\prime}\right) & \text { if } n<-1, \\ -\frac{1}{2} \alpha^{1 / 2} \cot \left(\pi / 2 c^{\prime}\right)+\frac{i}{2} \alpha^{1 / 2} & \text { if } n=-1, \\ -i \alpha^{-n-1 / 2} \psi\left(-1 / 2 c^{\prime}, 0,2 n+2\right) & \text { if } n \geq 0 .\end{cases}
$$

Proof. Put $r=1 / 2$ in Theorem 3.13 .

COROllary 3.16 .

$$
\alpha \sum_{k=1}^{\infty} \frac{2 k \cos \left(\pi k / c^{\prime}\right)}{e^{(\alpha-i \pi) 2 k / c^{\prime}}-1}=-\beta \sum_{k=1}^{\infty} \frac{k \cos \left(\pi k / c^{\prime}\right)}{\sinh \left((\beta+i \pi) k / c^{\prime}\right)}-\frac{c^{\prime}}{2} .
$$

Proof. Let $n=-1$ in Proposition 3.14 .

Corollary 3.17 .

$$
\begin{aligned}
\alpha^{1 / 2} \sum_{k=1}^{\infty} \frac{2 \sin \left(\pi k / c^{\prime}\right)}{e^{(\alpha-i \pi) 2 k / c^{\prime}}-1} & \\
& =(-\beta)^{1 / 2} \sum_{k=1}^{\infty} \frac{\sin \left(\pi k / c^{\prime}\right)}{\sinh \left((\beta+i \pi) k / c^{\prime}\right)}-\frac{1}{2} \alpha^{1 / 2} \cot \left(\frac{\pi}{2 c^{\prime}}\right) .
\end{aligned}
$$

Proof. Let $n=-1$ in Proposition 3.15 .

Corollary 3.18. Let $r$ be any real number with $0<r<1$. For any positive integer $M$,

$$
\begin{aligned}
\alpha^{M} \sum_{k=1}^{\infty} \frac{k^{2 M-1} \cos \left(2 \pi r k / c^{\prime}\right) \sin \left(2 \pi k / c^{\prime}\right)}{\cosh \left(2 \alpha k / c^{\prime}\right)-\cos \left(2 \pi k / c^{\prime}\right)} \\
=-(-\beta)^{M} \sum_{k=1}^{\infty} \frac{k^{2 M-1} \cosh \left(2 \beta r k / c^{\prime}\right) \sin \left(2 \pi k / c^{\prime}\right)}{\cosh \left(2 \beta k / c^{\prime}\right)-\cos \left(2 \pi k / c^{\prime}\right)} .
\end{aligned}
$$

Proof. Let $0<r<1$ and consider the imaginary parts in Theorem 3.12 in the case of $n=-M<0$. 
Corollary 3.19. Let $r$ be any real number with $0<r<1$. For any positive integer $M$,

$$
\begin{aligned}
\alpha^{M-1 / 2} \sum_{k=1}^{\infty} \frac{k^{2 M-2} \sin \left(2 \pi r k / c^{\prime}\right) \sin \left(2 \pi k / c^{\prime}\right)}{\cosh \left(2 \alpha k / c^{\prime}\right)-\cos \left(2 \pi k / c^{\prime}\right)} \\
\quad=(-1)^{M+1} \beta^{M-1 / 2} \sum_{k=1}^{\infty} \frac{k^{2 M-2} \sinh \left(2 \beta r k / c^{\prime}\right) \sin \left(2 \pi k / c^{\prime}\right)}{\cosh \left(2 \beta k / c^{\prime}\right)-\cos \left(2 \pi k / c^{\prime}\right)} .
\end{aligned}
$$

Proof. Let $0<r<1$ and consider the real parts in Theorem 3.13 in the case of $n=-M<0$.

3.3. Case of $N \mid r_{1}$ and $N \mid\left(r_{1}+r_{2}\right)$. By Theorem 2.1, for any integer $n$ and $z \in \mathbb{H}$ with $\operatorname{Re}(z)>0$, we have

$$
\begin{aligned}
z^{n} H_{N}(V \tau,-n ; r, 0)= & H_{N}(\tau,-n ; R, 0)+(2 \pi i)^{n} L_{N}(\tau,-n ; R, 0) \\
& +\lim _{s \rightarrow-n}\left(D_{1}\left(0, r_{2} / c, s\right)+D_{2}\left(0,-r_{1}-r_{2}+r_{2} / c, s\right)\right),
\end{aligned}
$$

where $D_{1}\left(0, r_{2} / c, s\right), D_{2}\left(0,-r_{1}-r_{2}+r_{2} / c, s\right)$ are defined in Section 2. By the given conditions on $r_{1}$ and $r_{2}$,

$$
\frac{\varrho_{N}}{N}=c^{\prime}\left\{-r_{1}-r_{2}+\frac{r_{2}}{N c^{\prime}}\right\}-\left(1-N c^{\prime}\right)\left\{\frac{r_{1}+r_{2}}{N}\right\}=c^{\prime}\left\{\frac{r_{2}}{N c^{\prime}}\right\} .
$$

Hence employing Lemmas 2.2 and 2.3 of $[8$ ] together with Remark 2.2, we find that, for any $z \in \mathbb{H}$,

$$
\begin{gathered}
z^{2 n} \sum_{k=1}^{\infty} \frac{2 \cos \left(2 \pi r_{2} k / c\right)}{k^{2 n+1}\left(e^{\frac{2 \pi i k}{c^{\prime}}\left(\frac{1}{z}-1\right)}-1\right)}=\sum_{k=1}^{\infty} \frac{2 \cos \left(2 \pi r_{2} k / c\right)}{k^{2 n+1}\left(e^{\frac{2 \pi i k}{c^{\prime}}(1-z)}-1\right)} \\
+(2 \pi i)^{2 n+1} \sum_{j=1}^{c^{\prime}} \sum_{k=0}^{2 n+2} \frac{B_{k}\left(j / c^{\prime}\right) \bar{B}_{2 n+2-k}\left(j / c^{\prime}+\left\{r_{2} / c\right\}\right)}{k !(2 n+2-k) !}(-z)^{k-1}+J_{3}(n),
\end{gathered}
$$

where if $c \nmid r_{2}$, then

$$
J_{3}(n):= \begin{cases}\left(1-z^{2 n}\right)(2 \pi i)^{2 n}(-2 n-1) ! \mathcal{Z}_{+}\left(-2 n,\left\{r_{2} / c\right\}\right) & \text { if } n<0, \\ -2 \pi i \bar{B}_{1}\left(r_{2} / c\right) & \text { if } n=0, \\ \psi\left(r_{2} / c, 0,1+2 n\right)-z^{2 n} \psi\left(-r_{2} / c, 0,1+2 n\right) & \text { if } n>0,\end{cases}
$$

and if $c \mid r_{2}$, then

$$
J_{3}(n):= \begin{cases}\left(1-z^{2 n}\right) \zeta(1+2 n) & \text { if } n<0 \\ \pi i-\log z & \text { if } n=0 \\ \left(1-z^{2 n}\right) \zeta(1+2 n) & \text { if } n>0\end{cases}
$$


For $c \nmid r_{2}$, we also deduce that, for any $z \in \mathbb{H}$,

$$
\begin{gathered}
z^{2 n+1} \sum_{k=1}^{\infty} \frac{2 i \sin \left(2 \pi r_{2} k / c\right)}{k^{2 n+2}\left(e^{\frac{2 \pi i k}{c^{\prime}}\left(\frac{1}{z}-1\right)}-1\right)}=\sum_{k=1}^{\infty} \frac{2 i \sin \left(2 \pi r_{2} k / c\right)}{k^{2 n+2}\left(e^{\frac{2 \pi i k}{c^{\prime}}(1-z)}-1\right)} \\
+(2 \pi i)^{2 n+2} \sum_{j=1}^{c^{\prime}} \sum_{k=0}^{2 n+3} \frac{B_{k}\left(j / c^{\prime}\right) \bar{B}_{2 n+3-k}\left(j / c^{\prime}+\left\{r_{2} / c\right\}\right)}{k !(2 n+3-k) !}(-z)^{k-1}+\mathcal{J}_{3}(n),
\end{gathered}
$$

where

$$
\mathcal{J}_{3}(n):= \begin{cases}\left(z^{2 n+1}-1\right)(2 \pi i)^{2 n+1}(-2 n-2) ! \mathcal{Z}_{-}\left(-2 n-1,\left\{r_{2} / c\right\}\right) & \text { if } n<-1 \\ \frac{1}{2 i}\left(z^{-1}-1\right) \cot \left(\pi r_{2} / c\right)-\frac{1}{2}\left(z^{-1}+1\right) & \text { if } n=-1 \\ \psi\left(r_{2} / c, 0,2+2 n\right)+z^{2 n+1} \psi\left(-r_{2} / c, 0,2+2 n\right) & \text { if } n \geq 0 .\end{cases}
$$

Theorem 3.20. Let $\alpha, \beta>0$ with $\alpha \beta=\pi^{2}$. Suppose that $r$ is an integer and $c^{\prime}$ is a positive integer with $c^{\prime} \nmid r$. For any integer $n$,

$$
\begin{aligned}
& \alpha^{-n} \sum_{k=1}^{\infty} \frac{2 \cos \left(2 \pi r k / c^{\prime}\right)}{k^{2 n+1}\left(e^{2 k(\alpha-i \pi) / c^{\prime}}-1\right)}=(-\beta)^{-n} \sum_{k=1}^{\infty} \frac{2 \cos \left(2 \pi r k / c^{\prime}\right)}{k^{2 n+1}\left(e^{2 k(\beta+i \pi) / c^{\prime}}-1\right)} \\
& \quad-2^{2 n+1} \sum_{j=1}^{c^{\prime}} \sum_{k=0}^{2 n+2} \frac{B_{k}\left(j / c^{\prime}\right) \bar{B}_{2 n+2-k}\left(j / c^{\prime}+\left\{r / c^{\prime}\right\}\right)}{k !(2 n+2-k) !} \alpha^{n-k+1}(-\pi i)^{k}+J_{4}(n),
\end{aligned}
$$

where

$$
J_{4}(n):= \begin{cases}2^{2 n}\left(\alpha^{n}-(-\beta)^{n}\right)(-2 n-1) ! \mathcal{Z}_{+}\left(-2 n,\left\{r / c^{\prime}\right\}\right) & \text { if } n<0, \\ -2 \pi i \bar{B}_{1}\left(r / c^{\prime}\right) & \text { if } n=0, \\ (-\beta)^{-n} \psi\left(r / c^{\prime}, 0,1+2 n\right)-\alpha^{-n} \psi\left(-r / c^{\prime}, 0,1+2 n\right) & \text { if } n>0 .\end{cases}
$$

Proof. Put $z=\pi i / \alpha$ and $r_{2} / N=r$ in (3.17).

Theorem 3.21. Let $\alpha, \beta>0$ with $\alpha \beta=\pi^{2}$. Suppose that $r$ is an integer and $c^{\prime}$ is a positive integer with $c^{\prime} \nmid r$. For any integer $n$,

$$
\begin{aligned}
& \alpha^{-n-1 / 2} \sum_{k=1}^{\infty} \frac{2 i \sin \left(2 \pi r k / c^{\prime}\right)}{k^{2 n+2}\left(e^{2 k(\alpha-i \pi) / c^{\prime}}-1\right)} \\
& =(-\beta)^{-n-1 / 2} \sum_{k=1}^{\infty} \frac{2 i \sin \left(2 \pi r k / c^{\prime}\right)}{k^{2 n+2}\left(e^{2 k(\beta+i \pi) / c^{\prime}}-1\right)} \\
& -2^{2 n+2} \sum_{j=1}^{c^{\prime}} \sum_{k=0}^{2 n+3} \frac{B_{k}\left(j / c^{\prime}\right) \bar{B}_{2 n+3-k}\left(j / c^{\prime}+\left\{r / c^{\prime}\right\}\right)}{k !(2 n+3-k) !}(-\pi i)^{k} \alpha^{n-k+3 / 2}+\mathcal{J}_{4}(n),
\end{aligned}
$$


where

$$
\mathcal{J}_{4}(n):= \begin{cases}2^{2 n+1}\left((-\beta)^{n+1 / 2}-\alpha^{n+1 / 2}\right)(-2 n-2) ! & \\ \quad \times \mathcal{Z}_{-}\left(-2 n-1,\left\{r / c^{\prime}\right\}\right) & \text { if } n<-1, \\ \frac{1}{2 i}\left(\alpha^{1 / 2}-(-\beta)^{1 / 2}\right) \cot \left(\pi r / c^{\prime}\right)-\frac{1}{2}\left(\alpha^{1 / 2}+(-\beta)^{1 / 2}\right) & \text { if } n=-1, \\ (-\beta)^{-n-1 / 2} \psi\left(r / c^{\prime}, 0,2+2 n\right) & \\ +\alpha^{-n-1 / 2} \psi\left(-r / c^{\prime}, 0,2+2 n\right) & \text { if } n \geq 0 .\end{cases}
$$

Proof. Put $z=\pi i / \alpha$ and $r_{2} / N=r$ in 3.18.

Corollary 3.22. For any positive integer $M$,

$$
\sum_{k=1}^{\infty} \frac{k^{4 M-1} \cos \left(2 \pi r k / c^{\prime}\right) \sin \left(2 \pi k / c^{\prime}\right)}{\cosh \left(2 \pi k / c^{\prime}\right)-\cos \left(2 \pi k / c^{\prime}\right)}=0 .
$$

Proof. Let $\alpha=\beta=\pi$ in Theorem 3.20. Replace $n$ by $-2 M$ for any positive integer $M$, and use

$$
\frac{1}{e^{x-i x}-1}-\frac{1}{e^{x+i x}-1}=\frac{i \sin (x)}{\cosh (x)-\cos (x)} .
$$

Corollary 3.23. For any positive integer $M$,

$$
\begin{aligned}
\sum_{k=1}^{\infty} \frac{\cos \left(2 \pi r k / c^{\prime}\right) \sin \left(2 \pi k / c^{\prime}\right)}{k^{4 M+1}\left(\cosh \left(2 \pi k / c^{\prime}\right)-\cos \left(2 \pi k / c^{\prime}\right)\right)} \\
=-\frac{(2 \pi)^{4 M+1}}{2} \sum_{j=1}^{c^{\prime}} \sum_{k=0}^{2 M} \frac{B_{2 k+1}\left(j / c^{\prime}\right) \bar{B}_{4 M+1-2 k}\left(j / c^{\prime}+\left\{r / c^{\prime}\right\}\right)}{(2 k+1) !(4 M+1-2 k) !}(-1)^{k+1} \\
\quad-\frac{(2 \pi)^{4 M+1}}{2(4 M+1) !} \bar{B}_{4 M+1}\left(\frac{r}{c^{\prime}}\right)
\end{aligned}
$$

Proof. Let $\alpha=\beta=\pi$ in Theorem 3.20 . Replace $n$ by $2 M$ for any positive integer $M$. For $n>0$, applying Corollary 3.47 of [8], we find that

$$
\begin{aligned}
\psi\left(r / c^{\prime}, 0,1+4 n\right)-\psi\left(-r / c^{\prime}, 0,1+4 n\right) & =2 i \sum_{k=1}^{\infty} \frac{\sin \left(2 \pi r k / c^{\prime}\right)}{k^{4 n+1}} \\
& =\frac{i(2 \pi)^{4 n+1}}{(4 n+1) !} \bar{B}_{4 n+1}\left(\frac{r}{c^{\prime}}\right) .
\end{aligned}
$$

Employing 3.19 and considering the imaginary parts, we arrive at the desired results.

Corollary 3.24. For any positive integer $M$,

$$
\sum_{j=1}^{c^{\prime}} \sum_{k=0}^{2 M-1} \frac{B_{2 k}\left(j / c^{\prime}\right) \bar{B}_{4 M-1-2 k}\left(j / c^{\prime}+\left\{r / c^{\prime}\right\}\right)}{(2 k) !(4 M-1-2 k) !}(-1)^{k}=0 .
$$


Proof. Let $\alpha=\beta=\pi$ in Theorem 3.20. Replace $n$ by $2 M-1$. Consider the imaginary parts.

Corollary 3.25 .

$$
\begin{aligned}
\alpha \sum_{k=1}^{\infty} \frac{k \cos \left(2 \pi r k / c^{\prime}\right)}{e^{\frac{2 k}{c^{\prime}}(\alpha-i \pi)}-1} & +\beta \sum_{k=1}^{\infty} \frac{k \cos \left(2 \pi r k / c^{\prime}\right)}{e^{\frac{2 k}{c^{\prime}}(\beta+i \pi)}-1} \\
& =\frac{\beta+\alpha}{4 \pi^{2}}\left(\zeta\left(2,\left\{r / c^{\prime}\right\}\right)+\zeta\left(2,1-\left\{r / c^{\prime}\right\}\right)\right)-c^{\prime} / 4 .
\end{aligned}
$$

Proof. Put $n=-1$ in Theorem 3.20 .

Corollary 3.26.

$$
\begin{aligned}
& \sum_{k=1}^{\infty} \frac{\cos \left(2 \pi r k / c^{\prime}\right) \sin \left(2 \pi k / c^{\prime}\right)}{k\left(\cosh \left(2 \pi k / c^{\prime}\right)-\right.}\left.\cos \left(2 \pi k / c^{\prime}\right)\right) \\
&=\pi \sum_{j=1}^{c^{\prime}} B_{1}\left(j / c^{\prime}\right) \bar{B}_{1}\left(j / c^{\prime}+\left\{r / c^{\prime}\right\}\right)-\pi \bar{B}_{1}\left(r / c^{\prime}\right) .
\end{aligned}
$$

Proof. Let $\alpha=\beta=\pi$ and $n=0$ in Theorem 3.20. Use 3.19.

Corollary 3.27. For $c^{\prime} \neq 1$,

$$
\sum_{k=1}^{\infty} \frac{\cos \left(2 \pi k / c^{\prime}\right) \sin \left(2 \pi k / c^{\prime}\right)}{k\left(\cosh \left(2 \pi k / c^{\prime}\right)-\cos \left(2 \pi k / c^{\prime}\right)\right)}=\frac{\left(c^{\prime}-2\right)\left(c^{\prime}+2\right) \pi}{6 c^{\prime}} .
$$

Proof. Let $r=1$ in Corollary 3.26 .

Corollary 3.28 .

$$
\begin{aligned}
& \sum_{k=1}^{\infty} \frac{\sin \left(2 \pi r k / c^{\prime}\right) \sin \left(2 \pi k / c^{\prime}\right)}{\cosh \left(2 \pi k / c^{\prime}\right)-\cos \left(2 \pi k / c^{\prime}\right)}=\sum_{k=1}^{\infty} \frac{\sin \left(2 \pi r k / c^{\prime}\right) \cos \left(2 \pi k / c^{\prime}\right)}{\cosh \left(2 \pi k / c^{\prime}\right)-\cos \left(2 \pi k / c^{\prime}\right)} \\
&-\sum_{k=1}^{\infty} \frac{\sin \left(2 \pi r k / c^{\prime}\right) e^{-2 \pi k / c^{\prime}}}{\cosh \left(2 \pi k / c^{\prime}\right)-\cos \left(2 \pi k / c^{\prime}\right)}+\frac{1}{2} \cot \left(\pi r / c^{\prime}\right)
\end{aligned}
$$

Proof. Let $n=-1$ in Theorem 3.21 and consider the imaginary parts.

Theorem 3.29. Let $\alpha, \beta>0$ with $\alpha \beta=\pi^{2}$. Suppose that $r$ is an integer and $c^{\prime}$ is a positive integer with $c^{\prime} \mid r$. For any integer $n$,

$$
\begin{gathered}
\alpha^{-n} \sum_{k=1}^{\infty} \frac{1}{k^{2 n+1}\left(e^{2 k(\alpha-i \pi) / c^{\prime}}-1\right)}=(-\beta)^{-n} \sum_{k=1}^{\infty} \frac{1}{k^{2 n+1}\left(e^{2 k(\beta+i \pi) / c^{\prime}}-1\right)} \\
-2^{2 n} \sum_{j=1}^{c^{\prime}} \sum_{k=0}^{2 n+2} \frac{B_{k}\left(j / c^{\prime}\right) \bar{B}_{2 n+2-k}\left(j / c^{\prime}\right)}{k !(2 n+2-k) !} \alpha^{n-k+1}(-\pi i)^{k}+\frac{1}{2} J_{4}^{\prime}(n)
\end{gathered}
$$


where

$$
J_{4}^{\prime}(n)= \begin{cases}\left((-\beta)^{-n}-\alpha^{-n}\right) \frac{B_{-2 n}}{2 n} & \text { if } n<0, \\ \frac{1}{2} \log \alpha-\frac{1}{2} \log \beta+\frac{\pi}{2} i & \text { if } n=0, \\ \left((-\beta)^{-n}-\alpha^{-n}\right) \zeta(1+2 n) & \text { if } n>0 .\end{cases}
$$

Proof. Put $z=\pi i / \alpha$ in (3.17). For $n<0$, use (2.5), i.e., $\zeta(1+2 n)=$ $B_{-2 n} /(2 n)$.

Corollary 3.30. For any positive integer $M$,

$$
\sum_{k=1}^{\infty} \frac{k^{4 M-1} \sin \left(2 \pi k / c^{\prime}\right)}{\cosh \left(2 \pi k / c^{\prime}\right)-\cos \left(2 \pi k / c^{\prime}\right)}=0
$$

Proof. Let $\alpha=\beta=\pi$ in Theorem 3.29 and replace $n$ by $-2 M$ for any positive integer $M$. Equate the real parts.

Corollary 3.31. For any positive integer $M$,

$$
\begin{aligned}
& \sum_{k=1}^{\infty} \frac{\sin \left(2 \pi k / c^{\prime}\right)}{k^{4 M+1} \cosh \left(2 \pi k / c^{\prime}\right)-\cos \left(2 \pi k / c^{\prime}\right)} \\
& \quad=-\frac{(2 \pi)^{4 M+1}}{2} \sum_{j=1}^{c^{\prime}} \sum_{k=0}^{2 M} \frac{B_{2 k+1}\left(j / c^{\prime}\right) \bar{B}_{4 M+1-2 k}\left(j / c^{\prime}\right)}{(2 k+1) !(4 M+1-2 k) !}(-1)^{k} .
\end{aligned}
$$

Proof. Let $\alpha=\beta=\pi$ in Theorem 3.29 and replace $n$ by $2 M$ for any positive integer $M$. Equate the real parts.

Corollary 3.32. For any positive integer $M$,

$$
\sum_{j=1}^{c^{\prime}} \sum_{k=0}^{2 M+1} \frac{B_{2 k}\left(j / c^{\prime}\right) \bar{B}_{4 M+2-2 k}\left(j / c^{\prime}\right)}{(2 k) !(4 n+2-2 k) !}(-1)^{k}=0 .
$$

Proof. Under the conditions in the proof of Corollary 3.31, equate the imaginary parts.

COROllary 3.33 .

$$
\alpha \sum_{k=1}^{\infty} \frac{k}{e^{2 k(\alpha-i \pi) / c^{\prime}}-1}+\beta \sum_{k=1}^{\infty} \frac{k}{e^{2 k(\beta+i \pi) / c^{\prime}}-1}=-\frac{c^{\prime}}{4}+\frac{1}{24}(\beta+\alpha) .
$$

Proof. Put $n=-1$ in Theorem 3.29 and use $\zeta(-1)=-1 / 12$.

Corollary 3.34 .

$\sum_{k=1}^{\infty} \frac{k \cos \left(2 \pi k / c^{\prime}\right)}{\cosh \left(2 \pi k / c^{\prime}\right)-\cos \left(2 \pi k / c^{\prime}\right)}=\sum_{k=1}^{\infty} \frac{k e^{-2 \pi k / c^{\prime}}}{\cosh \left(2 \pi k / c^{\prime}\right)-\cos \left(2 \pi k / c^{\prime}\right)}-\frac{c^{\prime}}{4 \pi}+\frac{1}{12}$.

Proof. Let $\alpha=\beta=\pi$ in Corollary 3.33. Use

$$
\frac{1}{e^{x-i x}-1}+\frac{1}{e^{x+i x}-1}=\frac{\cos (x)}{\cosh (x)-\cos (x)}-\frac{e^{-x}}{\cosh (x)-\cos (x)} .
$$


Corollary 3.35 .

$$
\begin{aligned}
\sum_{k=1}^{\infty} \frac{1}{k\left(e^{2 k(\alpha-i \pi) / c^{\prime}}-1\right)} & -\sum_{k=1}^{\infty} \frac{1}{k\left(e^{2 k(\beta+i \pi) / c^{\prime}}-1\right)} \\
& =\frac{1}{12 c^{\prime}}(\beta-\alpha)+\frac{1}{4} \log \frac{\alpha}{\beta}+\frac{\left(c^{\prime}-1\right)\left(c^{\prime}-2\right) \pi i}{12 c^{\prime}} .
\end{aligned}
$$

Proof. Put $n=0$ in Theorem 3.29 .

Corollary 3.36 .

$$
\sum_{k=1}^{\infty} \frac{\sin \left(2 \pi k / c^{\prime}\right)}{k\left(\cosh \left(2 \pi k / c^{\prime}\right)-\cos \left(2 \pi k / c^{\prime}\right)\right)}=\frac{\left(c^{\prime}-1\right)\left(c^{\prime}-2\right) \pi}{12 c^{\prime}} .
$$

Proof. Let $\alpha=\beta=\pi$ in Corollary 3.35 and apply (3.19).

COROLlary 3.37. For any positive integer $M$,

$$
\sum_{k=1}^{\infty} \frac{k^{4 M+1} \cos \left(2 \pi k / c^{\prime}\right)}{\cosh \left(2 \pi k / c^{\prime}\right)-\cos \left(2 \pi k / c^{\prime}\right)}=\sum_{k=1}^{\infty} \frac{k^{4 M+1} e^{-2 \pi k / c^{\prime}}}{\cosh \left(2 \pi k / c^{\prime}\right)-\cos \left(2 \pi k / c^{\prime}\right)}+\frac{B_{4 M+2}}{4 M+2} .
$$

Proof. Let $\alpha=\beta=\pi$ in Theorem 3.29 and replace $n$ by $-2 M-1$ for any positive integer $M$. Use 3.20.

COROLlary 3.38. For any positive integer $M$,

$$
\begin{aligned}
\sum_{k=1}^{\infty} \frac{\cos \left(2 \pi k / c^{\prime}\right)}{k^{4 M-1}\left(\cosh \left(2 \pi k / c^{\prime}\right)-\cos \left(2 \pi k / c^{\prime}\right)\right)} \\
=\sum_{k=1}^{\infty} \frac{e^{-2 \pi k / c^{\prime}}}{k^{4 M-1}\left(\cosh \left(2 \pi k / c^{\prime}\right)-\cos \left(2 \pi k / c^{\prime}\right)\right)} \\
\quad-\frac{(2 \pi)^{4 M-1}}{2} \sum_{j=1}^{c^{\prime}} \sum_{k=0}^{2 M} \frac{B_{2 k}\left(j / c^{\prime}\right) \bar{B}_{4 M-2 k}\left(j / c^{\prime}\right)}{(2 k) !(4 M-2 k) !}(-1)^{k}-\zeta(4 M-1) .
\end{aligned}
$$

Proof. Let $\alpha=\beta=\pi$ in Theorem 3.29 and replace $n$ by $2 M-1$. Equate the real parts.

Corollary 3.39 .

$$
\sum_{j=1}^{c^{\prime}} \sum_{k=0}^{2 M-1} \frac{B_{2 k+1}\left(j / c^{\prime}\right) \bar{B}_{4 M-1-2 k}\left(j / c^{\prime}\right)}{(2 k+1) !(4 M-1-2 k) !}(-1)^{k}=0 .
$$

Proof. Under the conditions in the proof of Corollary 3.38, equate the imaginary parts.

4. Case $c^{\prime}=2$. In this section, we assume $c^{\prime}=2$. Then using the results in Section 3 of [8], we obtain a number of infinite series identities, many of which are new. 
4.1. $N \nmid r_{1}$ and $N \nmid\left(r_{1}+r_{2}\right)$. Put $c^{\prime}=2$ in Theorem 3.1. It is easy to see that

$$
\begin{aligned}
\sum_{k=1}^{\infty} & \frac{\cosh \left(\left(2\left(r+r^{\prime}\right)-2[r]-1\right) \pi i k / 2+(1-2\{r\}) \alpha k / 2\right)}{k^{2 n+1} \sinh ((\alpha-i \pi) k / 2)} \\
= & 2^{-2 n-1} \sum_{k=1}^{\infty} \frac{\cosh \left(\left(r+r^{\prime}\right) 2 \pi i k+(1-2\{r\}) \alpha k\right)}{k^{2 n+1} \sinh (\alpha k)} \\
& +(-1)^{[r]} \sum_{k=0}^{\infty} \frac{\sinh \left(\left(2\left(r+r^{\prime}\right) \pi i+(1-2\{r\}) \alpha\right)(2 k+1) / 2\right)}{(2 k+1)^{2 n+1} \cosh (\alpha(2 k+1) / 2)}
\end{aligned}
$$

and

$$
\begin{aligned}
\sum_{k=1}^{\infty} & \frac{\cosh \left(\left(-2 r+2\left[r+r^{\prime}\right]+1\right) \pi i k / 2+\left(1-2\left\{r+r^{\prime}\right\}\right) \beta k / 2\right)}{k^{2 n+1} \sinh ((\beta+i \pi) k / 2)} \\
= & 2^{-2 n-1} \sum_{k=1}^{\infty} \frac{\cosh \left(-2 \pi i r k+\left(1-2\left\{r+r^{\prime}\right\}\right) \beta k\right)}{k^{2 n+1} \sinh (\beta k)} \\
& \quad+(-1)^{\left[r+r^{\prime}\right]} \sum_{k=0}^{\infty} \frac{\sinh \left(\left(-2 \pi i r+\left(1-2\left\{r+r^{\prime}\right\}\right) \beta\right)(2 k+1) / 2\right)}{(2 k+1)^{2 n+1} \cosh (\beta(2 k+1) / 2)}
\end{aligned}
$$

Theorem 4.1. Let $\alpha, \beta>0$ with $\alpha \beta=\pi^{2}$. Suppose that $r$, $r^{\prime}$ are real numbers such that $r, r+r^{\prime}$ are not integers. Then, for any integer $n$,

$$
\begin{aligned}
(-1)^{[r]} \alpha^{-n} & \sum_{k=0}^{\infty} \frac{\sinh \left(\left(2\left(r+r^{\prime}\right) \pi i+(1-2\{r\}) \alpha\right)(2 k+1) / 2\right)}{(2 k+1)^{2 n+1} \cosh (\alpha(2 k+1) / 2)} \\
= & (-1)^{\left[r+r^{\prime}\right]}(-\beta)^{-n} \sum_{k=0}^{\infty} \frac{\sinh \left(\left(-2 \pi i r+\left(1-2\left\{r+r^{\prime}\right\}\right) \beta\right)(2 k+1) / 2\right)}{(2 k+1)^{2 n+1} \cosh (\beta(2 k+1) / 2)} \\
& -2^{2 n+1} \sum_{j=1}^{2} \sum_{k=0}^{2 n+2} \frac{B_{k}\left(\frac{j-\left\{r+r^{\prime}\right\}}{2}\right) \bar{B}_{2 n+2-k}\left(\frac{j+\varrho_{N} / N}{2}\right)}{k !(2 n+2-k) !}(-\pi i)^{k} \alpha^{n-k+1} \\
& +\sum_{k=0}^{2 n+2} \frac{\bar{B}_{k}\left(r+r^{\prime}\right) \bar{B}_{2 n+2-k}\left(\varrho_{N} / N\right)}{k !(2 n+2-k) !}(\pi i)^{k} \alpha^{n-k+1} .
\end{aligned}
$$

Proof. Put 4.1 and 4.2 in the first equation in Theorem 3.1 and use Theorem 3.1 in 8 .

Similarly we obtain the following theorem.

TheOREM 4.2. Let $\alpha, \beta>0$ with $\alpha \beta=\pi^{2}$. Suppose that $r, r^{\prime}$ are real numbers such that $r, r+r^{\prime}$ are not integers. Then, for any integer $n$, 


$$
\begin{aligned}
(-1)^{[r]} \alpha^{-n-1 / 2} \sum_{k=0}^{\infty} \frac{\cosh \left(\left(2\left(r+r^{\prime}\right) \pi i+(1-2\{r\}) \alpha\right)(2 k+1) / 2\right)}{(2 k+1)^{2 n+2} \cosh (\alpha(2 k+1) / 2)} \\
=(-1)^{\left[r+r^{\prime}\right]}(-\beta)^{-n-1 / 2} \sum_{k=0}^{\infty} \frac{\cosh \left(\left(-2 \pi i r+\left(1-2\left\{r+r^{\prime}\right\}\right) \beta\right)(2 k+1) / 2\right)}{(2 k+1)^{2 n+2} \cosh (\beta(2 k+1) / 2)} \\
\quad-2^{2 n+2} \sum_{j=1}^{2} \sum_{k=0}^{2 n+3} \frac{B_{k}\left(\frac{j-\left\{r+r^{\prime}\right\}}{2}\right) \bar{B}_{2 n+3-k}\left(\frac{j+\varrho_{N} / N}{2}\right)}{k !(2 n+3-k) !}(-\pi i)^{k} \alpha^{n-k+3 / 2} \\
\quad+\sum_{k=0}^{2 n+3} \frac{\bar{B}_{k}\left(r+r^{\prime}\right) \bar{B}_{2 n+3-k}\left(\varrho_{N} / N\right)}{k !(2 n+3-k) !}(\pi i)^{k} \alpha^{n-k+3 / 2} .
\end{aligned}
$$

Corollary 4.3. For any positive integer $M$,

$$
\begin{aligned}
(-1)^{[r]} \alpha^{M} \sum_{k=0}^{\infty} \frac{\sinh \left(\left(2\left(r+r^{\prime}\right) \pi i+(1-2\{r\}) \alpha\right)(2 k+1) / 2\right)}{(2 k+1)^{1-2 M} \cosh (\alpha(2 k+1) / 2)} \\
=(-1)^{\left[r+r^{\prime}\right]}(-\beta)^{M} \sum_{k=0}^{\infty} \frac{\sinh \left(\left(-2 \pi i r+\left(1-2\left\{r+r^{\prime}\right\}\right) \beta\right)(2 k+1) / 2\right)}{(2 k+1)^{1-2 M} \cosh (\beta(2 k+1) / 2)} .
\end{aligned}
$$

Proof. Put $n=-M$ in Theorem 4.1.

Corollary 4.4.

$$
\begin{aligned}
& (-1)^{[r]} \sum_{k=0}^{\infty} \frac{\sinh \left(\left(2\left(r+r^{\prime}\right) \pi i+(1-2\{r\}) \alpha\right)(2 k+1) / 2\right)}{(2 k+1) \cosh (\alpha(2 k+1) / 2)} \\
& \quad=(-1)^{\left[r+r^{\prime}\right]} \sum_{k=0}^{\infty} \frac{\sinh \left(\left(-2 \pi i r+\left(1-2\left\{r+r^{\prime}\right\}\right) \beta\right)(2 k+1) / 2\right)}{(2 k-1) \cosh (\beta(2 k+1) / 2)}+A
\end{aligned}
$$

where

$$
A:= \begin{cases}\pi i / 4 & \text { if }\left[\varrho_{N} / N\right] \text { is odd, } \\ -\pi i / 4 & \text { if }\left[\varrho_{N} / N\right] \text { is even } .\end{cases}
$$

Proof. Put $n=0$ in Theorem 4.1.

Corollary 4.5. For any positive integer $M$,

$$
\begin{aligned}
& (-1)^{[r]} \alpha^{M-1 / 2} \sum_{k=0}^{\infty} \frac{\cosh \left(\left(2\left(r+r^{\prime}\right) \pi i+(1-2\{r\}) \alpha\right)(2 k+1) / 2\right)}{(2 k+1)^{2-2 M} \cosh (\alpha(2 k+1) / 2)} \\
& =(-1)^{\left[r+r^{\prime}\right]}(-\beta)^{M-1 / 2} \sum_{k=0}^{\infty} \frac{\cosh \left(\left(-2 \pi i r+\left(1-2\left\{r+r^{\prime}\right\}\right) \beta\right)(2 k+1) / 2\right)}{(2 k+1)^{2-2 M} \cosh (\beta(2 k+1) / 2)} .
\end{aligned}
$$

Proof. Let $n=-M$ in Theorem 4.2. If $M>1$, then Corollary 4.5 is obvious. If $M=1$, then we can check that the sums over the Bernoulli polynomials vanish. 
Let us assume that $r$ is a real number with $0<r<1$ and $r^{\prime}=0$. We see that

$$
\varrho_{N} / N=-2[N r]-r
$$

Thus

$$
\left[\varrho_{N} / N\right]=-2[-N r]-1, \quad\left\{\varrho_{N} / N\right\}=1-r .
$$

TheOREM 4.6. Let $\alpha, \beta>0$ with $\alpha \beta=\pi^{2}$. Let $r$ be a real number with $0<r<1$. Then, for any integer $n$,

$$
\begin{aligned}
\alpha^{-n} \sum_{k=0}^{\infty} \frac{\sinh ((2 \pi i r+\alpha(1-2 r))(2 k+1) / 2)}{(2 k+1)^{2 n+1} \cosh (\alpha(2 k+1) / 2)} \\
=(-\beta)^{-n} \sum_{k=0}^{\infty} \frac{\sinh ((-2 \pi i r+\beta(1-2 r))(2 k+1) / 2)}{(2 k+1)^{2 n+1} \cosh (\beta(2 k+1) / 2)} \\
\quad-2^{2 n+1} \sum_{j=1}^{2} \sum_{k=0}^{2 n+2} \frac{B_{k}\left(\frac{j-r}{2}\right) \bar{B}_{2 n+2-k}\left(\frac{j-r}{2}\right)}{k !(2 n+2-k) !}(-\pi i)^{k} \alpha^{n-k+1} \\
\quad+\sum_{k=0}^{2 n+2} \frac{B_{k}(r) B_{2 n+2-k}(r)}{k !(2 n+2-k) !}(-\pi i)^{k} \alpha^{n-k+1} .
\end{aligned}
$$

Proof. Let $r^{\prime}=0$ and $0<r<1$ in Theorem 4.1 and apply 4.3.

TheOREM 4.7. Let $\alpha, \beta>0$ with $\alpha \beta=\pi^{2}$. Let $r$ be a real number with $0<r<1$. Then, for any integer $n$,

$$
\begin{aligned}
\alpha^{-n-1 / 2} \sum_{k=0}^{\infty} \frac{\cosh ((2 \pi i r+\alpha(1-2 r))(2 k+1) / 2)}{(2 k+1)^{2 n+2} \cosh (\alpha(2 k+1) / 2)} \\
=(-\beta)^{-n-1 / 2} \sum_{k=0}^{\infty} \frac{\cosh ((-2 \pi i r+\beta(1-2 r))(2 k+1) / 2)}{(2 k+1)^{2 n+2} \cosh (\beta(2 k+1) / 2)} \\
-2^{2 n+2} \sum_{j=1}^{2} \sum_{k=0}^{2 n+3} \frac{B_{k}\left(\frac{j-r}{2}\right) \bar{B}_{2 n+3-k}\left(\frac{j-r}{2}\right)}{k !(2 n+3-k) !}(-\pi i)^{k} \alpha^{n-k+3 / 2} \\
-\sum_{k=0}^{2 n+3} \frac{B_{k}(r) \bar{B}_{2 n+3-k}(r)}{k !(2 n+3-k) !}(-\pi i)^{k} \alpha^{n-k+3 / 2} .
\end{aligned}
$$

Theorems 4.6 and 4.7 can be divided into two parts, i.e., the real parts and the imaginary parts. We use

$$
\begin{aligned}
& \sinh (x \pm i y)=\sinh x \cos y \pm i \cosh x \sin y \\
& \cosh (x \pm i y)=\cosh x \cos y \pm i \sinh x \sin y
\end{aligned}
$$

for any real numbers $x, y$. 
Theorem 4.8. Let $\alpha, \beta>0$ with $\alpha \beta=\pi^{2}$. Let $r$ be a real number with $0<r<1$. Then, for any integer $n$,

$$
\begin{aligned}
& \alpha^{-n} \sum_{k=0}^{\infty} \frac{\sinh (\alpha(1-2 r)(2 k+1) / 2) \cos (\pi r(2 k+1))}{(2 k+1)^{2 n+1} \cosh (\alpha(2 k+1) / 2)} \\
& =(-\beta)^{-n} \sum_{k=0}^{\infty} \frac{\sinh (\beta(1-2 r)(2 k+1) / 2) \cos (\pi r(2 k+1))}{(2 k+1)^{2 n+1} \cosh (\beta(2 k+1) / 2)} \\
& -2^{2 n+1} \sum_{j=1}^{2} \sum_{k=0}^{n+1} \frac{B_{2 k}\left(\frac{j-r}{2}\right) \bar{B}_{2 n+2-2 k}\left(\frac{j-r}{2}\right)}{(2 k) !(2 n+2-2 k) !} \alpha^{n-k+1}(-\beta)^{k} \\
& +\sum_{k=0}^{n+1} \frac{B_{2 k}(r) B_{2 n+2-2 k}(r)}{(2 k) !(2 n+2-2 k) !} \alpha^{n-k+1}(-\beta)^{k} .
\end{aligned}
$$

Proof. Equate the real parts in Theorem 4.6.

Theorem 4.9. Let $\alpha, \beta>0$ with $\alpha \beta=\pi^{2}$. Let $r$ be a real number with $0<r<1$. Then, for any integer $n$,

$$
\begin{aligned}
\alpha^{-n} \sum_{k=0}^{\infty} \frac{\cosh (\alpha(1-2 r)(2 k+1) / 2) \sin (\pi r(2 k+1))}{(2 k+1)^{2 n+1} \cosh (\alpha(2 k+1) / 2)} \\
=-(-\beta)^{-n} \sum_{k=0}^{\infty} \frac{\cosh (\beta(1-2 r)(2 k+1) / 2) \sin (\pi r(2 k+1))}{(2 k+1)^{2 n+1} \cosh (\beta(2 k+1) / 2)} \\
\quad+2^{2 n+1} \pi \sum_{j=1}^{2} \sum_{k=0}^{n} \frac{B_{2 k+1}\left(\frac{j-r}{2}\right) \bar{B}_{2 n+1-2 k}\left(\frac{j-r}{2}\right)}{(2 k+1) !(2 n+1-2 k) !} \alpha^{n-k}(-\beta)^{k} \\
-\pi \sum_{k=0}^{n} \frac{B_{2 k+1}(r) B_{2 n+1-2 k}(r)}{(2 k+1) !(2 n+1-2 k) !} \alpha^{n-k}(-\beta)^{k} .
\end{aligned}
$$

Proof. Equate the imaginary parts in Theorem 4.6.

TheOREM 4.10. Let $\alpha, \beta>0$ with $\alpha \beta=\pi^{2}$. Let $r$ be a real number with $0<r<1$. Then, for any integer $n$,

$$
\begin{aligned}
\alpha^{-n-1 / 2} & \sum_{k=0}^{\infty} \frac{\cosh (\alpha(1-2 r)(2 k+1) / 2) \cos (\pi r(2 k+1))}{(2 k+1)^{2 n+2} \cosh (\alpha(2 k+1) / 2)} \\
= & (-1)^{n+1} \beta^{-n-1 / 2} \sum_{k=0}^{\infty} \frac{\sinh (\beta(1-2 r)(2 k+1) / 2) \sin (\pi r(2 k+1))}{(2 k+1)^{2 n+2} \cosh (\beta(2 k+1) / 2)} \\
& -2^{2 n+2} \sum_{j=1}^{2} \sum_{k=0}^{n+1} \frac{B_{2 k}\left(\frac{j-r}{2}\right) \bar{B}_{2 n+3-2 k}\left(\frac{j-r}{2}\right)}{(2 k) !(2 n+3-2 k) !} \alpha^{n-k+3 / 2}(-\beta)^{k} \\
& -\sum_{k=0}^{n+1} \frac{B_{2 k}(r) \bar{B}_{2 n+3-2 k}(r)}{(2 k) !(2 n+3-2 k) !} \alpha^{n-k+3 / 2}(-\beta)^{k} .
\end{aligned}
$$

Proof. Equate the real parts in Theorem 4.7 . 
We see that the imaginary parts in Theorem 4.7 give the same theorem as Theorem 4.10 .

COROllary 4.11. For any positive integer $M$,

$$
\begin{aligned}
& \alpha^{2 M} \sum_{k=0}^{\infty} \frac{(2 k+1)^{4 M-1} \sinh (\alpha(1-2 r)(2 k+1) / 2) \cos (\pi r(2 k+1))}{\cosh (\alpha(2 k+1) / 2)} \\
&=\beta^{2 M} \sum_{k=0}^{\infty} \frac{(2 k+1)^{4 M-1} \sinh (\beta(1-2 r)(2 k+1) / 2) \cos (\pi r(2 k+1))}{\cosh (\beta(2 k+1) / 2)} .
\end{aligned}
$$

Proof. Put $n=-2 M$ in Theorem 4.8 .

COROllary 4.12. For any positive integer $M$,

$$
\begin{aligned}
\alpha^{2 M-1} & \sum_{k=0}^{\infty} \frac{(2 k+1)^{2 M-3} \cosh (\alpha(1-2 r)(2 k+1) / 2) \sin (\pi r(2 k+1))}{\cosh (\alpha(2 k+1) / 2)} \\
= & \beta^{2 M-1} \sum_{k=0}^{\infty} \frac{(2 k+1)^{2 M-3} \cosh (\beta(1-2 r)(2 k+1) / 2) \sin (\pi r(2 k+1))}{\cosh (\beta(2 k+1) / 2)} .
\end{aligned}
$$

Proof. Put $n=-2 M+1$ in Theorem 4.9 .

COROllary 4.13. For any positive integer $M$,

$$
\begin{aligned}
& \alpha^{2 M-3 / 2} \sum_{k=0}^{\infty} \frac{(2 k+1)^{4 M-4} \cosh (\alpha(1-2 r)(2 k+1) / 2) \cos (\pi r(2 k+1))}{\cosh (\alpha(2 k+1) / 2)} \\
&=\beta^{2 M-3 / 2} \sum_{k=0}^{\infty} \frac{(2 k+1)^{4 M-4} \sinh (\beta(1-2 r)(2 k+1) / 2) \sin (\pi r(2 k+1))}{\cosh (\beta(2 k+1) / 2)} .
\end{aligned}
$$

Proof. Put $n=-2 M+1$ in Theorem 4.10. The sums of Bernoulli polynomials vanish when $n=-1$.

Corollaries 4.114 .13 are symmetric with respect to $\alpha$ and $\beta$.

COROllary 4.14. For any positive integer $M$,

$$
\sum_{k=0}^{\infty} \frac{(2 k+1)^{4 M-3} \sinh (\pi(1-2 r)(2 k+1) / 2) \cos (\pi r(2 k+1))}{\cosh (\pi(2 k+1) / 2)}=0 .
$$

Proof. Replace $-n$ by $2 M-1$ and let $\alpha=\beta=\pi$ in Theorem 4.8 .

COROllary 4.15. For any positive integer $M$,

$$
\sum_{k=0}^{\infty} \frac{(2 k+1)^{4 M-1} \cosh (\pi(1-2 r)(2 k+1) / 2) \sin (\pi r(2 k+1))}{\cosh (\pi(2 k+1) / 2)}=0 .
$$

Proof. Replace $-n$ by $2 M$ and let $\alpha=\beta=\pi$ in Theorem 4.9. 
COROllary 4.16. For any positive integer $M$,

$$
\sum_{k=0}^{\infty} \frac{(2 k+1)^{4 M-2} \cosh (\pi(1-2 r)(2 k+1) / 2) \cos (\pi r(2 k+1))}{\cosh (\pi(2 k+1) / 2)}=0 .
$$

Proof. Replace $-n$ by $2 M$ and let $\alpha=\beta=\pi$ in Theorem 4.10 .

Corollary 4.17.

$$
\begin{aligned}
\sum_{k=0}^{\infty} \frac{\sinh (\alpha(1-2 r)(2 k+1) / 2) \cos (\pi r(2 k+1))}{(2 k+1)} \cosh (\alpha(2 k+1) / 2) \\
=\sum_{k=0}^{\infty} \frac{\sinh (\beta(1-2 r)(2 k+1) / 2) \cos (\pi r(2 k+1))}{(2 k+1) \cosh (\beta(2 k+1) / 2)} .
\end{aligned}
$$

Proof. Put $n=0$ in Theorem 4.8 .

Corollary 4.18.

$$
\sum_{k=0}^{\infty} \frac{\cosh (\pi(1-2 r)(2 k+1) / 2) \sin (\pi r(2 k+1))}{(2 k+1) \cosh (\pi(2 k+1) / 2)}=\frac{\pi}{8} .
$$

Proof. Put $n=0$ and let $\alpha=\beta=\pi$ in Theorem 4.9

COROllary 4.19. For any positive integer $M$,

$$
\begin{aligned}
\sum_{k=0}^{\infty} \frac{\sinh (\pi(1-2 r)(2 k+1) / 2) \cos (\pi r(2 k+1))}{(2 k+1)^{4 M-1} \cosh (\pi(2 k+1) / 2)} \\
=-\frac{(2 \pi)^{4 M-1}}{2} \sum_{j=1}^{2} \sum_{k=0}^{2 M} \frac{B_{2 k}\left(\frac{j-r}{2}\right) \bar{B}_{4 M-2 k}\left(\frac{j-r}{2}\right)}{(2 k) !(4 M-2 k) !}(-1)^{k} \\
+\frac{\pi^{4 M-1}}{2} \sum_{k=0}^{2 M} \frac{B_{2 k}(r) B_{4 M-2 k}(r)}{(2 k) !(4 M-2 k) !}(-1)^{k} .
\end{aligned}
$$

Proof. Put $n=2 M-1$ for any positive integer $M$ and let $\alpha=\beta=\pi$ in Theorem 4.8 .

COROllary 4.20. For any positive integer $M$,

$$
\begin{aligned}
\sum_{k=0}^{\infty} \frac{\cosh (\pi(1-2 r)(2 k+1) / 2) \sin (\pi r(2 k+1))}{(2 k+1)^{4 M+1} \cosh (\pi(2 k+1) / 2)} & \\
= & \frac{(2 \pi)^{4 M+1}}{2} \sum_{j=1}^{2} \sum_{k=0}^{2 M} \frac{B_{2 k+1}\left(\frac{j-r}{2}\right) \bar{B}_{4 M+1-2 k}\left(\frac{j-r}{2}\right)}{(2 k+1) !(4 M+1-2 k) !}(-1)^{k} \\
& -\frac{\pi^{4 M+1}}{2} \sum_{k=0}^{2 M} \frac{B_{2 k+1}(r) B_{4 M+1-2 k}(r)}{(2 k+1) !(4 M+1-2 k) !}(-1)^{k} .
\end{aligned}
$$


Proof. Put $n=2 M$ for any positive integer $M$ and let $\alpha=\beta=\pi$ in Theorem 4.9,

Remark 4.21. If we put $r=1 / 2$, then Theorem 4.9 yields Proposition 3.21 in [8], which is found in Ramanujan's Notebooks [11] and established by other authors [8, p. 13].

4.2. $N \mid r_{1}$ and $N \nmid\left(r_{1}+r_{2}\right)$. In this case, we see that

$$
\frac{\varrho_{N}}{N}=-2\left[-r_{2}+\frac{r_{2}}{2 N}\right]-2 N\left[\frac{r_{2}}{N}\right]+\left[\frac{r_{2}}{N}\right] .
$$

Thus $\left\{\varrho_{N} / N\right\}=0$. First, put $c^{\prime}=2$ in Theorem 3.12. It is easy to see that

$$
\begin{aligned}
& \sum_{k=1}^{\infty} \frac{\cos (\pi r k)}{k^{2 n+1}\left(e^{(\alpha-i \pi) k}-1\right)} \\
& \quad=\sum_{k=1}^{\infty} \frac{\cos (2 \pi r k)}{(2 k)^{2 n+1}\left(e^{2 \alpha k}-1\right)}-\sum_{k=0}^{\infty} \frac{\cos (\pi r(2 k+1))}{(2 k+1)^{2 n+1}\left(e^{\alpha(2 k+1)}+1\right)}
\end{aligned}
$$

and

$$
\begin{aligned}
& \sum_{k=1}^{\infty} \frac{\cosh ((2[r]+1) \pi i k / 2+(1-2\{r\}) \beta k / 2)}{k^{2 n+1} \sinh ((\beta+i \pi) k / 2)} \\
= & \sum_{k=1}^{\infty} \frac{\cosh ((1-2\{r\}) \beta k)}{(2 k)^{2 n+1} \sinh (\beta k)}+(-1)^{[r]} \sum_{k=0}^{\infty} \frac{\sinh ((1-2\{r\}) \beta(2 k+1) / 2)}{(2 k+1)^{2 n+1} \cosh (\beta(2 k+1) / 2)} .
\end{aligned}
$$

We divide the two sums of Bernoulli polynomials in Theorem 3.12 into the real and imaginary parts, namely,

$$
\begin{aligned}
\sum_{j=1}^{2} \sum_{k=0}^{2 n+2} & \frac{B_{k}\left(\frac{j-\{r\}}{2}\right) \bar{B}_{2 n+2-k}\left(\frac{j+[r]}{2}\right)}{k !(2 n+2-k) !} \alpha^{n-k+1}(-\pi i)^{k} \\
= & \sum_{j=1}^{2} \sum_{k=0}^{n+1} \frac{B_{2 k}\left(\frac{j-\{r\}}{2}\right) \bar{B}_{2 n+2-2 k}\left(\frac{j+[r]}{2}\right)}{(2 k) !(2 n+2-2 k) !} \alpha^{n-k+1}(-\beta)^{k} \\
& -\pi i \sum_{j=1}^{2} \sum_{k=0}^{n} \frac{B_{2 k+1}\left(\frac{j-\{r\}}{2}\right) \bar{B}_{2 n+1-2 k}\left(\frac{j+[r]}{2}\right)}{(2 k+1) !(2 n+1-2 k) !} \alpha^{n-k}(-\beta)^{k} .
\end{aligned}
$$

From the imaginary parts of 4.6,

$$
\begin{aligned}
\sum_{j=1}^{2} \sum_{k=0}^{n} \frac{B_{2 k+1}\left(\frac{j-\{r\}}{2}\right) \bar{B}_{2 n+1-2 k}\left(\frac{j+[r]}{2}\right)}{(2 k+1) !(2 n+1-2 k) !} & \alpha^{n-k}(-\beta)^{k} \\
= & -\frac{(-\beta)^{n}}{2(2 n+1) !} B_{2 n+1}\left(f_{r}\right),
\end{aligned}
$$


where

$$
f_{r}= \begin{cases}1-\{r\} / 2 & \text { if }[r] \text { is even } \\ 1 / 2-\{r\} / 2 & \text { if }[r] \text { is odd }\end{cases}
$$

In the case of $n=0$, we find that

$$
\frac{1}{2} \log \left(1-e^{-2 \pi i r}\right)-\log \left(1-e^{-\pi i r}\right)=\frac{1}{2} \log |\cot (\pi r / 2)|+\pi i \delta / 4,
$$

where

$$
\delta= \begin{cases}-1 & \text { if }[r] \equiv 0(\bmod 4) \\ -3 & \text { if }[r] \equiv 1(\bmod 4) \\ 3 & \text { if }[r] \equiv 2(\bmod 4) \\ 1 & \text { if }[r] \equiv 3(\bmod 4)\end{cases}
$$

For $n>0$,

$$
\begin{aligned}
\psi(-r / 2,0,2 n+1)=2^{-2 n-1} \psi(-r & , 0,2 n+1) \\
& +2^{-2 n-1} e^{-\pi i r} \psi(-r, 1 / 2,2 n+1) .
\end{aligned}
$$

In (4.9), the last term on the right hand side is expressed as

$$
\begin{aligned}
2^{-2 n-1} e^{-\pi i r} \psi(-r & , 1 / 2,2 n+1) \\
& =\sum_{k=0}^{\infty} \frac{\cos (\pi r(2 k+1))}{(2 k+1)^{2 n+1}}-i \sum_{k=0}^{\infty} \frac{\sin (\pi r(2 k+1))}{(2 k+1)^{2 n+1}} .
\end{aligned}
$$

Theorem 4.22. Let $\alpha, \beta>0$ with $\alpha \beta=\pi^{2}$. Let $r$ be any real number that is not an integer. Then, for any integer $n$,

$$
\begin{aligned}
\alpha^{-n} \sum_{k=0}^{\infty} & \frac{2 \cos (\pi r(2 k+1))}{(2 k+1)^{2 n+1}\left(e^{\alpha(2 k+1)}+1\right)} \\
= & (-1)^{[r]+1}(-\beta)^{-n} \sum_{k=0}^{\infty} \frac{\sinh (\beta(1-2\{r\})(2 k+1) / 2)}{(2 k+1)^{2 n+1} \cosh (\beta(2 k+1) / 2)} \\
& +2^{2 n+1} \sum_{j=1}^{2} \sum_{k=0}^{n+1} \frac{B_{2 k}\left(\frac{j-\{r\}}{2}\right) \bar{B}_{2 n+2-2 k}\left(\frac{j+[r]}{2}\right)}{(2 k) !(2 n+2-2 k) !} \alpha^{n-k+1}(-\beta)^{k} \\
& -\sum_{k=0}^{n+1} \frac{\bar{B}_{2 k}(r) B_{2 n+2-2 k}}{(2 k) !(2 n+2-2 k) !} \alpha^{n-k+1}(-\beta)^{k}+\frac{\pi i(-\beta)^{n} \bar{B}_{2 n+1}(r)}{2(2 n+1) !} \\
& +\frac{2^{2 n} \pi i(-\beta)^{n} B_{2 n+1}\left(f_{r}\right)}{(2 n+1) !}+I(n),
\end{aligned}
$$

where 


$$
I(n):= \begin{cases}2^{2 n}(-\beta)^{n}(-2 n-1) ! \mathcal{Z}_{+}(-2 n,\{r / 2\}) & \\ -2^{-1}(-\beta)^{n}(-2 n-1) ! \mathcal{Z}_{+}(-2 n,\{r\}) & \text { if } n<0, \\ \frac{1}{2} \log |\cot (\pi r / 2)|+\pi i \delta / 4 & \text { if } n=0, \\ \alpha^{-n}\left(\sum_{k=0}^{\infty} \frac{\cos (\pi r(2 k+1))}{(2 k+1)^{2 n+1}}-i \sum_{k=0}^{\infty} \frac{\sin (\pi r(2 k+1))}{(2 k+1)^{2 n+1}}\right) & \text { if } n>0,\end{cases}
$$

and $\mathcal{Z}_{+}(s, x), f_{r}, \delta$ are defined in (2.6), 4.7), 4.8, respectively.

Proof. Apply 4.4, (4.5) and Theorem 3.42 in [8] to Theorem 3.12, Employing (4.6)-4.10), we obtain the desired results.

If $0<r<1$, then the real parts of Theorem 4.22 can be compared with Theorem 4.17 in [5, p. 346].

Next, put $c^{\prime}=2$ in Theorem 3.13. Similarly we obtain the following theorem.

THEOREM 4.23. Let $\alpha, \beta>0$ with $\alpha \beta=\pi^{2}$. Let $r$ be any real number that is not an integer. Then, for any integer $n$,

$$
\begin{aligned}
& \alpha^{-n-1 / 2} \sum_{k=0}^{\infty} \frac{2 i \sin (\pi r(2 k+1))}{(2 k+1)^{2 n+2}\left(e^{\alpha(2 k+1)}+1\right)} \\
& =(-1)^{[r]+1}(-\beta)^{-n-1 / 2} \sum_{k=0}^{\infty} \frac{\cosh (\beta(1-2\{r\})(2 k+1) / 2)}{(2 k+1)^{2 n+2} \cosh (\beta(2 k+1) / 2)} \\
& \quad-2^{2 n+1} \pi i \sum_{j=1}^{2} \sum_{k=0}^{n+1} \frac{B_{2 k+1}\left(\frac{j-\{r\}}{2}\right) \bar{B}_{2 n+2-2 k}\left(\frac{j+[r]}{2}\right)}{(2 k+1) !(2 n+2-2 k) !} \alpha^{n-k+1 / 2}(-\beta)^{k} \\
& \quad-\pi i \sum_{k=0}^{n+1} \frac{\bar{B}_{2 k+1}(r) B_{2 n+2-2 k}}{(2 k+1) !(2 n+2-2 k) !} \alpha^{n-k+1 / 2}(-\beta)^{k} \\
& +\frac{(-1)^{n+1} \alpha^{1 / 2} \beta^{n+1} \bar{B}_{2 n+2}(r)}{2(2 n+2) !}+\frac{2^{2 n+1}(-1)^{n} \alpha^{1 / 2} \beta^{n+1} B_{2 n+2}\left(f_{r}\right)}{2(2 n+2) !}+\mathcal{I}(n),
\end{aligned}
$$

where

$$
\mathcal{I}(n):=\left\{\begin{aligned}
-2^{2 n+1}(-\beta)^{n+1 / 2}(-2 n-2) ! \mathcal{Z}_{-}(-2 n-1,\{r / 2\}) & \\
+2^{-1}(-\beta)^{n+1 / 2}(-2 n-2) ! \mathcal{Z}_{-}(-2 n-1,\{r\}) & \text { if } n<-1, \\
\frac{(-1)^{[r]} i}{2} \alpha^{1 / 2} \csc (\pi\{r\}) & \text { if } n=-1, \\
\alpha^{-n-1 / 2}\left(\sum_{k=0}^{\infty} \frac{\cos (\pi r(2 k+1))}{(2 k+1)^{2 n+2}}-i \sum_{k=0}^{\infty} \frac{\sin (\pi r(2 k+1))}{(2 k+1)^{2 n+2}}\right) & \text { if } n \geq 0,
\end{aligned}\right.
$$

and $\mathcal{Z}_{-}(s, x), f_{r}$ are defined in (2.6), 4.7), respectively.

If $0<r<1$, then the imaginary parts of Theorem 4.23 can be compared with Theorem 4.17 in [5, p. 347]. 
Corollary 4.24 .

$$
\begin{aligned}
\sum_{k=0}^{\infty} \frac{2 \cos (\pi r(2 k+1))}{(2 k+1)\left(e^{\alpha(2 k+1)}-1\right)}= & (-1)^{[r]+1} \sum_{k=0}^{\infty} \frac{\sinh ((1-2\{r\}) \beta(2 k+1) / 2)}{(2 k+1) \cosh (\beta(2 k+1) / 2)} \\
& +\frac{1}{2} \log \left|\cot \left(\frac{\pi r}{2}\right)\right|
\end{aligned}
$$

Proof. Let $n=0$ in Theorem 4.22 ,

COROLlary 4.25 .

$$
\begin{aligned}
\alpha^{1 / 2} \sum_{k=0}^{\infty} \frac{2 \sin (\pi r(2 k+1))}{e^{\alpha(2 k+1)}+1}= & (-1)^{[r]+1} \beta^{1 / 2} \sum_{k=0}^{\infty} \frac{\cosh (\beta(1-2\{r\})(2 k+1) / 2)}{\cosh (\beta(2 k+1) / 2)} \\
& +\frac{1}{2} \beta^{1 / 2}(1-\{r\})+\frac{(-1)^{[r]}}{2} \alpha^{1 / 2} \csc (\pi\{r\}) .
\end{aligned}
$$

Proof. Let $n=-1$ in Theorem 4.23 .

This last corollary is similar to Corollary 4.20 in [5, p. 349].

Proposition 4.26. Let $r$ be any real number that is not an integer. Then, for any positive integer $M$,

$$
\begin{aligned}
& \sum_{k=0}^{\infty} \frac{\sin (\pi r(2 k+1))}{(2 k+1)^{2 M-1}}=\frac{(-1)^{M-1} \pi^{2 M-1}}{2(2 M-1) !}\left(\bar{B}_{2 M-1}(r)+2^{2 M-1} B_{2 M-1}\left(f_{r}\right)\right), \\
& \sum_{k=0}^{\infty} \frac{\cos (\pi r(2 k+1))}{(2 k+1)^{2 M}}=\frac{(-1)^{M-1} \pi^{2 M}}{2(2 M) !}\left(\bar{B}_{2 M}(r)-2^{2 M-1} B_{2 M}\left(f_{r}\right)\right),
\end{aligned}
$$

where $f_{r}$ is defined in 4.7 .

Proof. Let $n=M-1$ and equate the imaginary parts and the real parts in Theorems 4.22 and 4.23 , respectively.

Proposition 4.26 should be compared with Corollary 3.47 in [8] or Theorem 3.2 in [4, p. 166].

Proposition 4.27. Let $\alpha, \beta>0$ with $\alpha \beta=\pi^{2}$. Then, for any integer $n$,

$$
\begin{aligned}
\alpha^{-n-1 / 2} & \sum_{k=0}^{\infty} \frac{2(-1)^{k}}{(2 k+1)^{2 n+2}\left(e^{\alpha(2 k+1)}+1\right)} \\
= & (-1)^{n} \beta^{-n-1 / 2} \sum_{k=0}^{\infty} \frac{\operatorname{sech}(\beta(2 k+1) / 2)}{(2 k+1)^{2 n+2}} \\
& -\pi \sum_{k=0}^{n+1} \frac{\left(2^{2 k}-2^{2 n+2}\right) B_{2 k+1}(1 / 4) B_{2 n+2-2 k}}{(2 k+1) !(2 n+2-2 k) !} \alpha^{n-k+1 / 2}(-\beta)^{k}+\mathcal{I}_{1}(n),
\end{aligned}
$$

where 


$$
\mathcal{I}_{1}(n):= \begin{cases}2^{-4 n-3} \alpha^{-n-1 / 2} B_{-2 n-1}(1 / 4) /(2 n+1) & \text { if } n<-1, \\ \frac{1}{2} \alpha^{1 / 2} & \text { if } n=-1, \\ -4^{-2 n-2} \alpha^{-n-1 / 2}(\zeta(2 n+2,1 / 4)-\zeta(2 n+2,3 / 4)) & \text { if } n \geq 0 .\end{cases}
$$

Proof. Put $r=1 / 2$ in Theorem 4.23. For $n<-1$, we see [8]

$$
\zeta(-2 n-1,1 / 4)-\zeta(-2 n-1,3 / 4)=\frac{(-1)^{n} 2^{-6 n-4} \pi^{-2 n-1} B_{-2 n-1}(1 / 4)}{(-2 n-1) !} .
$$

For the sums of Bernoulli polynomials, use [1]

$$
B_{n}(1 / 2)=-\left(1-2^{1-n}\right) B_{n}, \quad n \geq 0 .
$$

Proposition 4.27 is already given by Berndt [5, p. 348], in a different form with Euler polynomials $E_{n}(x), 0 \leq n<\infty$, under the assumption $h_{1}=h_{2}=1 / 2$, where $E_{n}(x)$ is defined by

$$
\frac{2 e^{x t}}{e^{t}+1}=\sum_{n=0}^{\infty} E_{n}(x) \frac{t^{n}}{n !} \quad(|t|<\pi) .
$$

COROllary 4.28 .

$$
\alpha^{1 / 2} \sum_{k=0}^{\infty} \frac{2(-1)^{k}}{e^{\alpha(2 k+1)}+1}-\frac{1}{2} \alpha^{1 / 2}=-\beta^{1 / 2} \sum_{k=0}^{\infty} \operatorname{sech}(\beta(2 k+1) / 2) .
$$

Proof. Let $n=-1$ in Proposition 4.27.

Berndt has also given a proof of this last corollary.

EXAMPLE 4.29. We obtain a class of interesting infinite series identities from Theorem 4.22 or 4.23 for special values of $r$. For example, let $r=1 / 4$ in Theorem 4.22, Then, using

$$
\cos (\pi(2 k+1) / 4)= \begin{cases}1 / \sqrt{2} & \text { if } k \equiv 0,3(\bmod 4) \\ -1 / \sqrt{2} & \text { if } k \equiv 1,2(\bmod 4)\end{cases}
$$

we have, for any positive integer $M$,

$$
\begin{aligned}
& \sqrt{2} \alpha^{M} \sum_{k \equiv 0,3(\bmod 4)} \frac{(2 k+1)^{2 M-1}}{e^{\alpha(2 k+1)}-1}-\sqrt{2} \alpha^{M} \sum_{k \equiv 1,2(\bmod 4)} \frac{(2 k+1)^{2 M-1}}{e^{\alpha(2 k+1)}-1} \\
& =-(-\beta)^{M} \sum_{k=0}^{\infty} \frac{(2 k+1)^{2 M-1} \sinh (\beta(2 k+1) / 4)}{\cosh (\beta(2 k+1) / 2)} \\
& -\left(2^{6 M-2}-2^{4 M-2}\right)\left|B_{2 M}\right| \pi^{2 M}(-\beta)^{-M}+2^{-2 M}(2 M) !(-\beta)^{-M} \mathcal{Z}_{+}(2 M, 1 / 8), \\
& \sum_{k \equiv 0,3(\bmod 4)} \frac{1}{(2 k+1)\left(e^{\alpha(2 k+1)}-1\right)}-\sum_{k \equiv 1,2(\bmod 4)} \frac{1}{(2 k+1)\left(e^{\alpha(2 k+1)}-1\right)} \\
& =-\frac{1}{2 \sqrt{2}} \sum_{k=0}^{\infty} \frac{\sinh (\beta(2 k+1) / 4)}{(2 k+1) \cosh (\beta(2 k+1) / 2)}+\frac{1}{2} \log (1+\sqrt{2}),
\end{aligned}
$$


and

$$
\begin{aligned}
\alpha^{-M} \sum_{k \equiv 0,3(\bmod 4)} \frac{(2 k+1)^{-2 M-1}}{e^{\alpha(2 k+1)}-1}-\alpha^{-M} \sum_{k \equiv 1,2(\bmod 4)} \frac{(2 k+1)^{-2 M-1}}{e^{\alpha(2 k+1)}-1} \\
=-\frac{(-\beta)^{-M}}{\sqrt{2}} \sum_{k=0}^{\infty} \frac{\sinh (\beta(2 k+1) / 4)}{(2 k+1)^{2 M+1} \cosh (\beta(2 k+1) / 2)} \\
+\frac{2^{2 M+1}}{\sqrt{2}} \sum_{k=0}^{M+1} \mathcal{B}_{M, 2 k}(3 / 8,1 / 2) \alpha^{M-k+1}(-\beta)^{k} \\
+\frac{2^{2 M+1}}{\sqrt{2}} \sum_{k=0}^{M+1} \mathcal{B}_{M, 2 k}(1 / 8,0) \alpha^{M-k+1}(-\beta)^{k} \\
\\
-\frac{1}{\sqrt{2}} \sum_{k=0}^{M+1} \mathcal{B}_{M, 2 k}(1 / 4,0) \alpha^{M-k+1}(-\beta)^{k} \\
+2^{-2 M-2} \alpha^{-M}\left(\mathcal{Z}_{+}(2 M, 1 / 8)-\mathcal{Z}_{+}(2 M, 3 / 8)\right)
\end{aligned}
$$

where

$$
\mathcal{B}_{n, k}(x, y):=\frac{B_{k}(x) B_{2 n+2-k}(y)}{k !(2 n+2-k) !} \quad \text { and } \quad \mathcal{B}_{n, k}(x):=\mathcal{B}_{n, k}(x, x) .
$$

4.3. $N \mid r_{1}$ and $N \mid\left(r_{1}+r_{2}\right)$. Suppose that $r$ is even in Theorem 3.29 Since

$$
\begin{aligned}
\sum_{k=1}^{\infty} \frac{1}{k^{2 n+1}\left(e^{(\alpha-i \pi) k}-1\right)}= & \sum_{k=1}^{\infty} \frac{2^{-2 n-1}}{k^{2 n+1}\left(e^{2 \alpha k}-1\right)} \\
& -\sum_{k=0}^{\infty} \frac{1}{(2 k+1)^{2 n+1}\left(e^{\alpha(2 k+1)}+1\right)}
\end{aligned}
$$

using Theorem 3.72 in [8], we find that

$$
\begin{aligned}
& \alpha^{-n} \sum_{k=0}^{\infty} \frac{1}{(2 k+1)^{2 n+1}\left(e^{\alpha(2 k+1)}+1\right)} \\
= & (-\beta)^{-n} \sum_{k=0}^{\infty} \frac{1}{(2 k+1)^{2 n+1}\left(e^{\beta(2 k+1)}+1\right)} \\
+ & 2^{2 n} \sum_{j=1}^{2} \sum_{k=0}^{2 n+2} \frac{B_{k}(j / 2) \bar{B}_{2 n+2-k}(j / 2)}{k !(2 n+2-k) !}(-\pi i)^{k} \alpha^{n-k+1} \\
& -\frac{1}{2} \sum_{k=0}^{n+1} \frac{B_{2 k} B_{2 n+2-2 k}}{(2 k) !(2 n+2-2 k) !} \alpha^{n-k+1}(-\beta)^{k}+2^{-2 n-2} K(n)-\frac{1}{2} J_{4}^{\prime}(n),
\end{aligned}
$$


where

$$
K(n):= \begin{cases}\left((-\beta)^{-n}-\alpha^{-n}\right) \frac{B_{-2 n}}{2 n} & \text { if } n<0 \\ \frac{1}{2} \log \alpha-\frac{1}{2} \log \beta & \text { if } n=0 \\ \left((-\beta)^{-n}-\alpha^{-n}\right) \zeta(1+2 n) & \text { if } n>0\end{cases}
$$

and $J_{4}^{\prime}(n)$ is defined in Theorem 3.29 .

TheOREM 4.30. Let $\alpha, \beta>0$ with $\alpha \beta=\pi^{2}$. Then, for any integer $n$,

$$
\begin{aligned}
\alpha^{-n} \sum_{k=0}^{\infty} & \frac{1}{(2 k+1)^{2 n+1}\left(e^{\alpha(2 k+1)}+1\right)} \\
= & (-\beta)^{-n} \sum_{k=0}^{\infty} \frac{1}{(2 k+1)^{2 n+1}\left(e^{\beta(2 k+1)}+1\right)} \\
& +\sum_{k=0}^{n+1} \frac{\left(2^{2 n+1}-2^{2 k-1}\right)\left(1-2^{-2 k}\right) B_{2 k} B_{2 n+2-2 k}}{(2 k) !(2 n+2-2 k) !} \alpha^{n-k+1}(-\beta)^{k}+I_{1}(n),
\end{aligned}
$$

where

$$
I_{1}(n):= \begin{cases}\left(2^{-2 n-2}-2^{-1}\right)\left((-\beta)^{-n}-\alpha^{-n}\right) \frac{B_{-2 n}}{2 n} & \text { if } n<0, \\ \left(2^{-2}-2^{-1}\right)\left(\frac{1}{2} \log \alpha-\frac{1}{2} \log \beta\right) & \text { if } n=0 \\ \left(2^{-2 n-2}-2^{-1}\right)\left((-\beta)^{-n}-\alpha^{-n}\right) \zeta(2 n+1) & \text { if } n>0 .\end{cases}
$$

Proof. In 4.11, the imaginary parts of the sums of Bernoulli polynomials can be expressed as

$$
-\pi i \sum_{j=1}^{2} \sum_{k=0}^{n} \frac{B_{2 k+1}(j / 2) \bar{B}_{2 n+1-2 k}(j / 2)}{(2 k+1) !(2 n+1-2 k) !} \alpha^{n-k}(-\beta)^{k}= \begin{cases}\pi i / 4 & \text { if } n=0 \\ 0 & \text { if } n>0\end{cases}
$$

Use the definition of $J_{4}^{\prime}(n)$ in Theorem 3.29 and apply the formula (see [1])

$$
B_{n}(1 / 2)=-\left(1-2^{1-n}\right) B_{n}, \quad n \geq 0 .
$$

Then the desired results follow.

If $n \neq 0$, then Theorem 4.30 has been first stated by Malukar 9 . Berndt [5] has also proved Theorem 4.30 for $n \neq 0$. In the case of $n<0$, Theorem 4.30 has been proved by Rao and Ayyar [12] and Nanjundiah [10].

COROLlary 4.31.

$$
\alpha \sum_{k=0}^{\infty} \frac{2 k+1}{e^{\alpha(2 k+1)}+1}=-\beta \sum_{k=0}^{\infty} \frac{2 k+1}{e^{\beta(2 k+1)}+1}+\frac{1}{24}(\beta+\alpha) .
$$

Proof. Let $n=-1$ in Theorem 4.30. Use $\zeta(-1)=-1 / 12$.

Corollary 4.32 .

$$
\sum_{k=0}^{\infty} \frac{2 k+1}{e^{\pi(2 k+1)}+1}=\frac{1}{24}
$$


Proof. Let $\alpha=\beta=\pi$ in Corollary 4.31 .

COROllary 4.33. For any positive integer $M$,

$$
\sum_{k=0}^{\infty} \frac{(2 k+1)^{4 M+1}}{e^{\pi(2 k+1)}+1}=\frac{B_{4 M+2}}{8 M+4}\left(2^{4 M+1}-1\right) .
$$

Proof. Put $n=-2 M-1$ for any positive integer $M$ and let $\alpha=\beta=\pi$ in Theorem 4.30,

Corollary 4.34 .

$\sum_{k=0}^{\infty} \frac{1}{(2 k+1)\left(e^{\alpha(2 k+1)}+1\right)}+\frac{1}{8} \log \alpha=\sum_{k=0}^{\infty} \frac{1}{(2 k+1)\left(e^{\beta(2 k+1)}+1\right)}+\frac{1}{8} \log \beta$.

Proof. Put $n=0$ in Theorem 4.30 .

Corollary 4.35. For any positive integer $M$,

$$
\begin{aligned}
\sum_{k=0}^{\infty} \frac{1}{(2 k+1)^{4 M-1}\left(e^{\pi(2 k+1)}+1\right)} & \\
= & \pi^{4 M-1} \sum_{k=0}^{2 M} \frac{\left(2^{4 M-2}-2^{2 k-2}\right)\left(1-2^{-2 k}\right) B_{2 k} B_{4 M-2 k}}{(2 k) !(4 M-2 k) !}(-1)^{k} \\
& -\left(2^{-4 M}-2^{-1}\right) \zeta(4 M-1) .
\end{aligned}
$$

Proof. Put $n=2 M-1$ for any positive integer and let $\alpha=\beta=\pi$ in Theorem 4.30,

Corollary 4.33 has already been found by Glaisher [6], Sandham [13] and Berndt [5]. Corollary 4.34 has also been established by Nanjundiah [10] and Berndt [5]. Berndt has also proved Corollary 4.35.

REMARK 4.36. The conclusion of Theorem 3.29 for $r$ odd coincides with Theorem 4.30. Proposition 4.27 is the case $c^{\prime}=4$ and $r$ odd of Theorem 3.21.

Acknowledgements. The author is pleased to thank B. C. Berndt for his helpful suggestions and comments throughout this work. The author also thanks the referee for his detailed comments.

This work was supported by the Korea Research Foundation Grant funded by the Korean Government (MOEHRD).(KRF-2006-214-C00003). This work also partially supported by BK21-Postech CoDiMaRo.

\section{References}

[1] M. Abramowitz and I. A. Stegun (eds.), Handbook of Mathematical Functions with Formulas, Graphs, and Mathematical Tables, Washington, DC, 1964.

[2] B. C. Berndt, Two new proofs of Lerch's functional equation, Proc. Amer. Math. Soc. 32 (1972), 403-408. 
[3] B. C. Berndt, Generalized Eisenstein series and modified Dedekind sums, J. Reine Angew. Math. 272 (1975), 182-193.

[4] —, Modular transformations and generalizations of several formulae of Ramanujan, Rocky Mountain J. Math. 7 (1977), 147-189.

[5] - Analytic Eisenstein series, theta-functions, and series relations in the spirit of Ramanujan, J. Reine Angew. Math. 304 (1978), 332-365.

[6] J. W. L. Glaisher, On the series which represent the twelve elliptic and four zeta functions, Mess. Math. 18 (1889), 1-84.

[7] J. Havil, Gamma: Exploring Euler's Constant, Princeton Univ. Press, Princeton, NJ, 2003.

[8] S. Lim, A few classes of infinite series from the generalized Eisenstein series, preprint.

[9] S. L. Malurkar, On the application of Herr Mellin's integrals to some series, J. Indian Math. Soc. 16 (1925-1926), 130-138.

[10] T. S. Nanjundiah, Certain summations due to Ramanujan, and their generalizations, Proc. Indian Acad. Sci. Sect. A 34 (1951), 215-228.

[11] S. Ramanujan, Notebooks of Srinivasa Ramanujan, 2 volumes, Tata Inst. Fund. Res., Bombay, 1957.

[12] M. B. Rao and M. V. Ayyar, On some infinite series and products, Part I, J. Indian Math. Soc. 15 (1923-1924), 150-162.

[13] H. F. Sandham, Some infinite series, Proc. Amer. Math. Soc. 5 (1954), 430-436.

\section{Sung-Geun Lim}

Department of Mathematics

Pohang University of Science and Technology

San 31, Hyoja-dong, Namgu

Pohang, Kyungbuk 790-784, Republic of Korea

E-mail: sglim@postech.ac.kr

Received on 1.12.2008

and in revised form on 23.7.2009 